MATHEMATICS OF COMPUTATION

Volume 78, Number 266, April 2009, Pages 815-844

S $0025-5718(08) 02197-2$

Article electronically published on November 20, 2008

\title{
ON SPECTRAL APPROXIMATIONS IN ELLIPTICAL GEOMETRIES USING MATHIEU FUNCTIONS
}

\author{
JIE SHEN AND LI-LIAN WANG
}

\begin{abstract}
We consider in this paper approximation properties and applications of Mathieu functions. A first set of optimal error estimates are derived for the approximation of periodic functions by using angular Mathieu functions. These approximation results are applied to study the Mathieu-Legendre approximation to the modified Helmholtz equation and Helmholtz equation. Illustrative numerical results consistent with the theoretical analysis are also presented.
\end{abstract}

\section{INTRODUCTION}

The Mathieu functions were first introduced by Mathieu in the nineteenth century, when he determined the vibrational modes of a stretched membrane with an elliptic boundary [27]. Since then, these functions have been extensively used in many areas of physics and engineering (cf., for instance, [25, 23, 19, 4, 21, 5, 32, 8, 17]), and many efforts have also been devoted to analytic, numerical and various other aspects of Mathieu functions (cf., for instance, [26, 6, 3, 36]). A considerable amount of mathematical results of Mathieu functions are contained in the books [28, 29, 1 . However, most of these results are concerned with classical properties such as identities, recursions and asymptotics. To the best of our knowledge, there are essentially no results on their approximation properties (in Sobolev spaces) which are necessary for the analysis of spectral methods using Mathieu functions. A main objective of this paper is to derive a first set of optimal approximation results for the Mathieu functions. These approximation results will be the basic ingredients for the numerical analysis of Mathieu approximations to partial differential equations.

Given a PDE in an elliptic or elliptic cylindrical geometry, a spectral method can be developed using two different approaches. In the first approach, we express the equation in the elliptic coordinates and then apply a Fourier approximation in the periodic direction combined with a polynomial approximation in nonperiodic direction(s), see, e.g., 9, 30. However, unlike in the polar and spherical geometries, all Fourier components are usually coupled together by the nonconstant coefficients in the transformed equation (only the Poisson equation in elliptical geometries can be decoupled (see [24]) where a mixed Fourier and finite difference solver was

Received by the editor March 4, 2008.

2000 Mathematics Subject Classification. Primary 65N35, 65N22, 65F05, 35J05.

Key words and phrases. Mathieu functions, elliptic coordinates, approximation in Sobolev spaces, Helmholtz equations.

The work of the first author was partially supported by NSF Grant DMS-0610646.

The work of the second author was partially supported by a Start-Up grant from NTU, Singapore MOE Grant T207B2202, and Singapore NRF2007IDM-IDM002-010.

(C)2008 American Mathematical Society 815

Reverts to public domain 28 years from publication 
proposed for this very particular case). The second approach is to use a Mathieu expansion in the periodic direction which leads to a dimension reduction, as in the polar and spherical geometries. In this paper, we will explore the second approach, derive optimal approximation results for the Mathieu expansions and apply them to study the Mathieu spectral method for the modified Helmholtz equation and the Helmholtz equation.

The rest of the paper is organized as follows. We first review some properties of the Mathieu functions in Section 2. The approximations by Mathieu functions in Sobolev spaces are studied in Sections 3 and 4. The applications of Mathieu approximations in spectral methods for two model Helmholtz-type equations are presented in Sections 5 and 6 . Section 7 is devoted to some numerical results and discussions.

We now introduce some notation to be used throughout this paper. Let $\Omega$ be a bounded, open domain in $\mathbb{R}^{d}, d=1,2,3$, and let $\varpi$ be a generic real weight function defined in $\Omega$. We denote by $L_{\varpi}^{2}(\Omega)$ a Hilbert space of real- or complexvalued functions with inner product and norm

$$
(u, v)_{\varpi}=\int_{\Omega} u \bar{v} \varpi d \Omega, \quad\|u\|_{\varpi}=(u, u)_{\varpi}^{1 / 2},
$$

where $\bar{v}$ is the complex conjugate of $v$. The weighted Sobolev spaces $H_{\varpi}^{s}(\Omega)(s=$ $0,1,2, \cdots)$ can be defined as usual with inner products, norms and semi-norms denoted by $(\cdot, \cdot)_{s, \varpi},\|\cdot\|_{s, \varpi}$ and $|\cdot|_{s, \varpi}$, respectively. For real $s>0, H_{\varpi}^{s}(\Omega)$ is defined by space interpolation as in 2 . The subscript $\varpi$ will be omitted from the notation in cases of $\varpi=1$. In particular, we have $L_{\varpi}^{2}(\Omega)=H_{\varpi}^{0}(\Omega)$. We will use $\|\cdot\|$ or $\|\cdot\|_{0}$ to denote the usual $L^{2}$-norm, and use $\|\cdot\|_{0, \varpi}$ or $\|\cdot\|_{L_{\varpi}^{2}(\Omega)}$ to denote the $\varpi$-weighted $L^{2}$-norm. We will use $\partial_{x}^{k}$ to denote the ordinary derivative $\frac{d^{k}}{d x^{k}}$, whenever no confusion may arise.

We will also use Sobolev spaces involving periodic functions. Namely, for $m>0$, we denote by $H_{p}^{m}(0,2 \pi)$ the subspace of $H^{m}(0,2 \pi)$ consisting of functions whose derivatives of order up to $m-1$ are $2 \pi$-periodic.

For any nonnegative integer $N$, let $P_{N}$ be the set of all algebraic polynomials of degree $\leq N$. We denote by $C$ a generic positive constant independent of any function, domain size and discretization parameters. We use the expression $A \lesssim B$ to mean that there exists a generic positive constant $C$ such that $A \leq C B$.

\section{Mathieu functions}

We recall that under the elliptic transform:

$$
x=c \cosh \mu \cos \theta, \quad y=c \sinh \mu \sin \theta
$$

(where $2 c$ is the focal distance), the two-dimensional Helmholtz equation in Cartesian coordinates

$$
\Delta U+k^{2} U=0
$$

becomes

$$
\frac{1}{\frac{c^{2}}{2}(\cosh (2 \mu)-\cos (2 \theta))}\left[\frac{\partial^{2} V}{\partial \mu^{2}}+\frac{\partial^{2} V}{\partial \theta^{2}}\right]+k^{2} V=0,
$$

where $V(\mu, \theta)=U(x, y)$. The Mathieu functions arise from applying the separation of variables approach in solving (2.3). More precisely, setting $V(\mu, \theta)=R(\mu) \Phi(\theta)$, 
we find that $\Phi(\theta)$ satisfies the (angular) Mathieu equation

$$
\frac{d^{2} \Phi}{d \theta^{2}}+(a-2 q \cos 2 \theta) \Phi=0
$$

and $R(\mu)$ satisfies the radial (or modified) Mathieu equation

$$
\frac{d^{2} R}{d \mu^{2}}-(a-2 q \cosh 2 \mu) R=0,
$$

where $a$ is the separation constant, and the parameter $q=c^{2} k^{2} / 4$. We note that the radial Mathieu equation (2.5) can be transformed to the Mathieu equation (2.4), and vice versa, via the mapping $\theta= \pm \mathrm{i} \mu$ (where $\mathrm{i}=\sqrt{-1}$ ).

The Mathieu equation (2.4) supplemented with a periodic boundary condition admits two families of linearly independent periodic solutions (eigenfunctions), namely the even and the odd Mathieu functions of order $m$ :

$$
\Phi_{m}(\theta ; q)=\operatorname{ce}_{m}(\theta ; q) \text { or } \operatorname{se}_{m+1}(\theta ; q), \quad m=0,1, \cdots,
$$

with the corresponding eigenvalues classified into two categories: even and odd, denoted by $a_{m}(q)$ and $b_{m}(q)$ for $\mathrm{ce}_{m}$ and $\mathrm{se}_{m}$, respectively. The notation ce and se, an abbreviation of "cosine-elliptic" and "sine-elliptic", were first introduced in [7. Notice that the Mathieu equation (2.4) becomes a harmonic equation when $q=0$, so the Mathieu functions reduce to the trigonometric functions, namely

$$
\operatorname{ce}_{m}(\theta ; 0)=\cos (m \theta), \quad \operatorname{se}_{m}(\theta ; 0)=\sin (m \theta), \quad a_{m}(0)=b_{m}(0)=m^{2} .
$$

The set of Mathieu functions $\left\{\mathrm{ce}_{m}, \mathrm{se}_{m+1}\right\}_{m=0}^{\infty}$ forms a complete orthogonal system in $L^{2}(0,2 \pi)$, and they are normalized so that

$$
\int_{0}^{2 \pi} \operatorname{ce}_{m}(\theta ; q) \operatorname{ce}_{n}(\theta ; q) d \theta=\int_{0}^{2 \pi} \operatorname{se}_{m}(\theta ; q) \operatorname{se}_{n}(\theta ; q) d \theta= \begin{cases}\pi, & \text { if } m=n \\ 0, & \text { if } m \neq n\end{cases}
$$

and

$$
\int_{0}^{2 \pi} \operatorname{ce}_{m}(\theta ; q) \operatorname{se}_{n}(\theta ; q) d \theta=0
$$

The parity, periodicity and normalization of the Mathieu functions are exactly the same as their trigonometric counterparts cos and $\sin$, namely, $\mathrm{ce}_{m}$ is even and $\mathrm{se}_{m}$ is odd, and they have period $\pi$ when $m$ is even, or period $2 \pi$ when $m$ is odd. Thanks to the periodicity and parity, the Mathieu functions can be expanded in the Fourier series

$$
\operatorname{ce}_{n}(\theta ; q)=\sum_{j=0}^{\infty} A_{j}^{(n)} \cos j \theta, \quad \operatorname{se}_{n}(\theta ; q)=\sum_{j=1}^{\infty} B_{j}^{(n)} \sin j \theta
$$

where the coefficients $\left\{A_{j}^{(n)}, B_{j}^{(n)}\right\}$ satisfy some five-term recursive relations (see Appendix A). This motivates most of the algorithms for computing Mathieu functions and their eigenvalues; see, e.g., [28, 1, 26, 36, 5, 3. . A package of Fortran and Matlab programs for manipulating Mathieu functions is also available (cf. [33]).

For the Helmholtz equation (2.2), the wave number $k>0$ so the parameter $q=c^{2} k^{2} / 4$ in the Mathieu equation is positive. However, for imaginary $k$ in (2.2), $q$ in (2.4) (2.5) becomes negative and equation (2.2) becomes elliptic. Hence, the Mathieu functions with $q>0$ are mostly used in applications involving wave equations, while the Mathieu functions with $q<0$ are useful in solving elliptic and parabolic equations [32, 8, 18. We see that with a change of variable $\theta \rightarrow \pi / 2-\theta$, 
the Mathieu equation (2.4) is transformed into an equation of the same type with $q<0$. Thus, the Mathieu functions with $q<0$ can be defined as follows (see, e.g., [28, 29, 1]):

$$
\begin{array}{ll}
\operatorname{ce}_{2 m}(\theta ;-q)=(-1)^{m} \operatorname{ce}_{2 m}(\pi / 2-\theta ; q), & a_{2 m}(-q)=a_{2 m}(q), \\
\operatorname{ce}_{2 m+1}(\theta ;-q)=(-1)^{m} \operatorname{se}_{2 m+1}(\pi / 2-\theta ; q), & a_{2 m+1}(-q)=b_{2 m+1}(q), \\
\operatorname{se}_{2 m+1}(\theta ;-q)=(-1)^{m} \operatorname{ce}_{2 m+1}(\pi / 2-\theta ; q), & b_{2 m+1}(-q)=a_{2 m+1}(q), \\
\operatorname{se}_{2 m+2}(\theta ;-q)=(-1)^{m} \operatorname{se}_{2 m+2}(\pi / 2-\theta ; q), & b_{2 m+2}(-q)=b_{2 m+2}(q) .
\end{array}
$$

While there have been quite a few papers/books devoted to analytic, numerical and various other aspects of Mathieu functions (cf., for instance, 26, 6, 3, 36]), there are essentially no results on the approximation of periodic functions using Mathieu functions (in Sobolev spaces). Such results are necessary for spectral methods for elliptic geometries.

\section{Mathieu approximations With $q>0$}

In this section, we consider approximation of periodic functions by Mathieu functions, and establish basic approximation results for Mathieu expansions. For clarity, we first consider the Mathieu functions with $q>0$, and the case with $q<0$ will be presented in the proceeding section.

To simplify the presentation, we shall use a uniform notation introduced in 29 to denote the odd and even Mathieu functions:

$$
\mathrm{me}_{2 m}(\theta ; q)=\mathrm{ce}_{m}(\theta ; q), \quad \mathrm{me}_{2 m+1}(\theta ; q)=\operatorname{se}_{m+1}(\theta ; q), \quad m=0,1, \cdots
$$

$$
\lambda_{2 m}(q)=a_{m}(q)+2 q, \quad \lambda_{2 m+1}(q)=b_{m}(q)+2 q, \quad m=0,1, \cdots .
$$

By (2.4), the eigenpairs $\left\{\left(\lambda_{m}, \mathrm{me}_{m}\right)\right\}$ satisfy the Sturm-Liouville equation:

$$
\mathcal{A}_{q} \mathrm{me}_{m}=\lambda_{m} \mathrm{me}_{m}, \quad m=0,1, \cdots,
$$

where the operator

$$
\mathcal{A}_{q} v=\left(-\partial_{\theta}^{2}+4 q \cos ^{2} \theta\right) v .
$$

Let $I:=(0,2 \pi)$. One verifies that the Sturm-Liouville operator $\mathcal{A}_{q}$ is compact, symmetric, strictly positive and self-adjoint in the sense that

$$
\begin{aligned}
& \left(\mathcal{A}_{q} u, v\right)=\left(v, \mathcal{A}_{q} u\right)=\left(u, \mathcal{A}_{q} v\right), \quad \forall u, v \in H_{p}^{2}(I), \\
& \left(\mathcal{A}_{q} v, v\right)=\left\|\partial_{\theta} v\right\|^{2}+4 q\|v\|_{0, \omega}^{2}>0, \quad \forall v \in H_{p}^{2}(I) \text { with } v \neq 0,
\end{aligned}
$$

where $\omega=\cos ^{2} \theta$. Hence, we infer from the standard Sturm-Liouville theory (see, e.g., [11]) that:

- The eigenvalues are all real, positive and distinct, i.e.,

$$
0<\lambda_{0}(q)<\lambda_{1}(q)<\cdots<\lambda_{m}(q)<\cdots .
$$

- The eigenfunctions, $\left\{\mathrm{me}_{m}\right\}_{m=0}^{\infty}$, form a complete orthogonal system in $L^{2}(I)$, and they are normalized so that

$$
\frac{1}{\pi} \int_{0}^{2 \pi} \mathrm{me}_{m}(\theta ; q) \mathrm{me}_{n}(\theta ; q) d \theta=\delta_{m n},
$$

where $\delta_{m n}$ is the Kronecker delta. 
- The Mathieu function $m_{m}$ has exactly $m$ zeros, which are real, distinct and located in $[0,2 \pi]$.

The following estimate for the eigenvalues is useful in the sequel.

Lemma 3.1. For any $q>0$, we have that

$$
m^{2}<\lambda_{m}(q)<m^{2}+4 q, \quad m=0,1, \cdots .
$$

Proof. Differentiating the equation (3.3) with respect to $q$ leads to

$$
\left(\mathcal{A}_{q}-\lambda_{m}\right) \frac{\partial \mathrm{me}_{m}}{\partial q}-\left(\lambda_{m}^{\prime}(q)-4 \cos ^{2} \theta\right) \mathrm{me}_{m}=0 .
$$

Multiplying the above equation by $\mathrm{me}_{m}$ and integrating the resulting equation over $(0,2 \pi)$, we deduce from the self-adjoint property of the operator $\mathcal{A}_{q}-\lambda_{m}$ that

$$
\begin{aligned}
0 & =\int_{0}^{2 \pi}\left[\left(\mathcal{A}_{q}-\lambda_{m}\right) \frac{\partial \mathrm{me}_{m}}{\partial q}\right] \mathrm{me}_{m} d \theta-\int_{0}^{2 \pi}\left(\lambda_{m}^{\prime}(q)-4 \cos ^{2} \theta\right) \mathrm{me}_{m}^{2} d \theta \\
& =\int_{0}^{2 \pi}\left[\left(\mathcal{A}_{q}-\lambda_{m}\right) \mathrm{me}_{m}\right] \frac{\partial \mathrm{me}_{m}}{\partial q} d \theta-\int_{0}^{2 \pi}\left(\lambda_{m}^{\prime}(q)-4 \cos ^{2} \theta\right) \mathrm{me}_{m}^{2} d \theta
\end{aligned}
$$

Therefore, by (3.3), the first term vanishes, and thanks to the orthogonality (3.7),

$$
\lambda_{m}^{\prime}(q)=\frac{4}{\pi} \int_{0}^{2 \pi} \cos ^{2} \theta \mathrm{me}_{m}^{2}(\theta ; q) d \theta .
$$

Since $0 \leq \cos ^{2} \theta \leq 1$, the above identity implies that

$$
0<\lambda_{m}^{\prime}(q)<4 \quad \Rightarrow \quad 0<\lambda_{m}(q)-\lambda_{m}(0)<4 q .
$$

Since $\lambda_{m}(0)=m^{2}$, the desired result (3.8) follows.

3.1. Inverse inequalities. We first point out that the Mathieu functions are orthogonal in $H_{p}^{1}(I)$ equipped with the inner product

$$
a_{q}(u, v)=\left(\partial_{\theta} u, \partial_{\theta} v\right)+4 q(u, v)_{\omega}, \quad \omega=\cos ^{2} \theta .
$$

Indeed, by integration by parts, (3.3) and (3.7),

$$
a_{q}\left(\mathrm{me}_{m}, \mathrm{me}_{n}\right)=\left(\mathcal{A}_{q} \mathrm{me}_{m}, \mathrm{me}_{n}\right)=\lambda_{m}\left(\mathrm{me}_{m}, \mathrm{me}_{n}\right)=\pi \lambda_{m} \delta_{m n}
$$

For any integer $M \geq 0$, we define the $(M+1)$-dimensional space

$$
X_{M}^{q}=\operatorname{span}\left\{\mathrm{me}_{m}: 0 \leq m \leq M\right\} .
$$

Thanks to the orthogonality (3.11), we are able to prove the following inequalities.

Lemma 3.2. For any $\phi, \psi \in X_{M}^{q}$, we have

$$
\left|a_{q}(\phi, \psi)\right| \leq \lambda_{M}\|\phi\|\|\psi\| \leq\left(M^{2}+4 q\right)\|\phi\|\|\psi\|,
$$

and the following inverse inequality holds

$$
\left\|\partial_{\theta}^{l} \phi\right\| \leq(M+c k)^{l}\|\phi\|, \quad l \geq 1,
$$

where $c k=\sqrt{4 q}$. 
Proof. For any $\phi, \psi \in X_{M}^{q}$, we write

$$
\phi=\sum_{m=0}^{M} \hat{\phi}_{m} \mathrm{me}_{m}, \quad \psi=\sum_{n=0}^{M} \hat{\psi}_{n} \mathrm{me}_{n} .
$$

By the orthogonality (3.7), we have

$$
\|\phi\|^{2}=\pi \sum_{m=0}^{M}\left|\hat{\phi}_{m}\right|^{2}, \quad\|\psi\|^{2}=\pi \sum_{n=0}^{M}\left|\hat{\psi}_{n}\right|^{2} .
$$

Using the orthogonality (3.11), the Cauchy-Schwarz inequality and Lemma 3.1 yields

$$
\begin{aligned}
\left|a_{q}(\phi, \psi)\right| & =\left|\sum_{m=0}^{M} \sum_{n=0}^{M} \hat{\phi}_{m} \hat{\psi}_{n} a_{q}\left(\mathrm{me}_{m}, \mathrm{me}_{n}\right)\right|=\left|\sum_{m=0}^{M} \pi \lambda_{m} \hat{\phi}_{m} \hat{\psi}_{m}\right| \\
& \leq \max _{0 \leq m \leq M}\left\{\lambda_{m}\right\}\left(\pi \sum_{m=0}^{M}\left|\hat{\phi}_{m}\right|^{2}\right)^{1 / 2}\left(\pi \sum_{m=0}^{M}\left|\hat{\psi}_{m}\right|^{2}\right)^{1 / 2} \\
& \leq \lambda_{M}\|\phi\|\|\psi\| \leq\left(M^{2}+4 q\right)\|\phi\|\|\psi\| .
\end{aligned}
$$

Thus, by the definition (3.10),

$$
a_{q}(\phi, \phi)=\left\|\partial_{\theta} \phi\right\|^{2}+4 q\|\phi\|_{0, \omega}^{2} \leq\left(M^{2}+c^{2} k^{2}\right)\|\phi\|^{2},
$$

which implies (3.14) with $l=1$. The desired result with $l>1$ can be derived by induction.

Remark 3.1. Compared with the Fourier basis, the factor $O(M)$ in the inverse inequality is optimal.

3.2. Error estimations of the $L^{2}$-projection. For any $v \in L^{2}(I)$, we write

$$
v(\theta)=\sum_{m=0}^{\infty} \hat{v}_{m} \mathrm{me}_{m}(\theta ; q)
$$

where

$$
\hat{v}_{m}=\hat{v}_{m}(q)=\frac{1}{\pi} \int_{0}^{2 \pi} v(\theta) \mathrm{me}_{m}(\theta ; q) d \theta, \quad m \geq 0 .
$$

Consider the orthogonal projection $\pi_{M}^{q}: L^{2}(I) \rightarrow X_{M}^{q}$ such that

$$
\left(\pi_{M}^{q} v-v, v_{M}\right)=0, \quad \forall v_{M} \in X_{M}^{q},
$$

or equivalently,

$$
\left(\pi_{M}^{q} v\right)(\theta)=\sum_{m=0}^{M} \hat{v}_{m} \mathrm{me}_{m}(\theta ; q) .
$$

To measure the projection error, we first introduce a Hilbert space associated with the Sturm-Liouville operator defined in (3.4). Since $\mathcal{A}_{q}$ is a compact, symmetric and (strictly) positive self-adjoint operator, the fractional power $\mathcal{A}_{q}^{1 / 2}$ is well defined, and there holds (see, e.g., Chapter II of [35]):

$$
\left\|\mathcal{A}_{q}^{1 / 2} v\right\|^{2}=a_{q}(v, v)
$$


where $a_{q}(\cdot, \cdot)$ is the inner product of $H_{p}^{1}(I)$ given in (3.10). Hence, for any integer $s \geq 0$, we define the Hilbert space

$$
\widetilde{H}_{q p}^{s}(I):=\left\{v \in H_{p}^{s}(I):\|v\|_{\widetilde{H}_{q p}^{s}(I)}^{2}:=\left\|\mathcal{A}_{q}^{s / 2} v\right\|^{2}=\left(\mathcal{A}_{q}^{s / 2} v, \mathcal{A}_{q}^{s / 2} v\right)<\infty\right\} .
$$

For real $s>0$, the space $\widetilde{H}_{q p}^{s}(I)$ and its norm are defined by space interpolation as in $[2]$.

Formally, one verifies by using (3.3), (3.7) and (3.11) that for any integer $n \geq 0$,

$$
\left\|\mathcal{A}_{q}^{n} v\right\|^{2}=\pi \sum_{m=0}^{\infty} \lambda_{m}^{2 n}\left|\hat{v}_{m}\right|^{2}, \quad\left\|\mathcal{A}_{q}^{n+1 / 2} v\right\|^{2}=a_{q}\left(\mathcal{A}_{q}^{n} v, \mathcal{A}_{q}^{n} v\right)=\pi \sum_{m=0}^{\infty} \lambda_{m}^{2 n+1}\left|\hat{v}_{m}\right|^{2} .
$$

Therefore, as with the Fourier expansion, the norm of the space $\widetilde{H}_{q p}^{s}(I)$ with real $s \geq 0$ can be characterized in the frequency space

$$
\|v\|_{\widetilde{H}_{q p}^{s}(I)}=\left\|\mathcal{A}_{q}^{s / 2} v\right\|=\left(\pi \sum_{m=0}^{\infty} \lambda_{m}^{s}\left|\hat{v}_{m}\right|^{2}\right)^{1 / 2} .
$$

We have the following fundamental approximation result.

Theorem 3.1. For any $v \in \widetilde{H}_{q p}^{s}(I)$ with $s \geq 0$,

$$
\left\|\pi_{M}^{q} v-v\right\| \leq \lambda_{M+1}^{-s / 2}\|v\|_{\widetilde{H}_{q p}^{s}(I)} .
$$

Proof. We only need to prove this result with integer $s \geq 0$. The general case can then be derived by space interpolation.

We first assume that $s=2 n$. By (3.16) and (3.3),

$$
\begin{aligned}
\hat{v}_{m} & =\frac{1}{\pi} \int_{0}^{2 \pi} v(\theta) \mathrm{me}_{m}(\theta ; q) d \theta=\frac{1}{\pi \lambda_{m}^{n}} \int_{0}^{2 \pi} v(\theta)\left(\mathcal{A}_{q}^{n} \mathrm{me}_{m}\right) d \theta \\
& =\frac{1}{\pi \lambda_{m}^{n}} \int_{0}^{2 \pi}\left(\mathcal{A}_{q}^{n} v\right)(\theta) \mathrm{me}_{m}(\theta ; q) d \theta=\frac{1}{\lambda_{m}^{n}} \widehat{\left(\mathcal{A}_{q}^{n} v\right)_{m}},
\end{aligned}
$$

where $\left\{\widehat{\left(\mathcal{A}_{q}^{n} v\right)}{ }_{m}\right\}$ are the coefficients of the Mathieu expansion of $\mathcal{A}_{q}^{n} v$. Therefore,

$$
\begin{aligned}
\left\|\pi_{M}^{q} v-v\right\|^{2} & =\pi \sum_{m=M+1}^{\infty}\left|\hat{v}_{m}\right|^{2}=\pi \sum_{m=M+1}^{\infty} \lambda_{m}^{-2 n}\left|\widehat{\left(A_{q}^{n} v\right)_{m}}\right|^{2} \\
& \leq \max _{m>M}\left\{\lambda_{m}^{-2 n}\right\}\left\|\mathcal{A}_{q}^{n} v\right\|^{2} \stackrel{(3.6)}{=} \lambda_{M+1}^{-s}\|v\|_{\widetilde{H}_{q p}^{s}(I)}^{2},
\end{aligned}
$$

which implies (3.23) with $s=2 n$.

We now prove (3.23) with $s=2 n+1$. By (3.21) and (3.6),

$$
\begin{aligned}
\left\|\pi_{M}^{q} v-v\right\|^{2} & =\pi \sum_{m=M+1}^{\infty}\left|\hat{v}_{m}\right|^{2} \leq \max _{m>M}\left\{\lambda_{m}^{-2 n-1}\right\} \sum_{m=M+1}^{\infty} \pi \lambda_{m}^{2 n+1}\left|\hat{v}_{m}\right|^{2} \\
& \leq \lambda_{M+1}^{-2 n-1} a_{q}\left(\mathcal{A}_{q}^{n} u, \mathcal{A}_{q}^{n} u\right) \leq \lambda_{M+1}^{-s}\|v\|_{\widetilde{H}_{q p}^{s}(I)}^{2} .
\end{aligned}
$$

This completes the proof.

The norm in the upper bound of the estimate (3.23) is expressed in the frequency space, and implicitly depends on the parameter $q$. To extract more explicit 
information from (3.23), we shall explore the explicit dependence of the approximation errors on the parameter $q=c^{2} k^{2} / 4$, and express the norm in terms of the derivatives of $v$. It is worthwhile to point out that setting $q=0$ in Theorem 3.1. we recover the classical Fourier approximation results. So, without loss of generality, we assume that the wave number

$$
k \geq k_{0}>0 \Rightarrow q \geq q_{0}=c^{2} k_{0}^{2} / 4>0 .
$$

It can be shown by induction that for any nonnegative integer $n$,

$$
\mathcal{A}_{q}^{n} v=(-1)^{n} \partial_{\theta}^{2 n} v+\sum_{j=1}^{n} p_{j}(\theta ; q) \partial_{\theta}^{2 n-2 j} v+\sum_{j=1}^{n-1} \tilde{p}_{j}(\theta ; q) \partial_{\theta}^{2 n-2 j-1} v,
$$

where $p_{j}(\theta ; q)$ and $\tilde{p}_{j}(\theta ; q)$ are polynomials of $4 q$ of degree $j$ (without constant term, i.e., $\left.p_{j}(\theta ; 0)=\tilde{p}_{j}(\theta ; 0)=0\right)$ with coefficients being polynomials of $\sin 2 \theta$ or $\cos 2 \theta$. We conclude that $\mathcal{A}_{q}^{n}$ is a continuous mapping from $H_{p}^{2 n}(I)$ to $L^{2}(I)$, since

$$
\left\|\mathcal{A}_{q}^{n} v\right\| \leq\left\|\partial_{\theta}^{2 n} v\right\|+C\left[\sum_{j=1}^{n-1} q^{j}\left(\left\|\partial_{\theta}^{2 n-2 j} v\right\|+\left\|\partial_{\theta}^{2 n-2 j-1} v\right\|\right)+q^{n}\|v\|\right] .
$$

Similarly, using (3.19) and (3.28) - 3.29) leads to

$$
\begin{aligned}
& \left\|\mathcal{A}_{q}^{n+1 / 2} v\right\|=\left(a_{q}\left(\mathcal{A}_{q}^{n} v, \mathcal{A}_{q}^{n} v\right)\right)^{1 / 2} \leq\left\|\partial_{\theta}\left(\mathcal{A}_{q}^{n} v\right)\right\|+2 \sqrt{q}\left\|\mathcal{A}_{q}^{n} v\right\|_{0, \omega} \\
& \quad \leq\left\|\partial_{\theta}^{2 n+1} v\right\|+C\left[\sum_{j=1}^{n-1} q^{j+1 / 2}\left(\left\|\partial_{\theta}^{2 n-2 j} v\right\|+\left\|\partial_{\theta}^{2 n-2 j+1} v\right\|\right)+q^{n+1 / 2}\|v\|\right],
\end{aligned}
$$

where we recall from (3.10) that $\omega=\cos ^{2} \theta$. As a consequence, we have the embedding relation $H_{p}^{s}(I) \subset \widetilde{H}_{q p}^{s}(I)$, and

$$
\begin{aligned}
&\|v\|_{\widetilde{H}_{q p}^{s}(I)}=\left\|\mathcal{A}_{q}^{s / 2} v\right\| \leq|v|_{s}+C \sum_{j=0}^{s-2} q^{(s-j) / 2}|v|_{j} \lesssim(1+q)^{s / 2}\|v\|_{s}, \quad s \geq 2 \\
&\|v\|_{\widetilde{H}_{q p}^{s}(I)} \leq|v|_{s}+2 \sqrt{q}\|v\|_{0, \omega}, \quad s=1,2 .
\end{aligned}
$$

Notice that the powers of $q$ for derivatives of different order are different; such an explicit estimate is necessary when the wave number $k \gg 1$ (see Remark [3.2).

The following estimate is a direct consequence of Theorem 3.1 and (3.31).

Corollary 3.1. For any $v \in H_{p}^{s}(I)$ with $s \geq 0$,

$$
\left\|\pi_{M}^{q} v-v\right\| \leq M^{-s}\left(|v|_{s}+C \sum_{j=0}^{s-2} q^{(s-j) / 2}|v|_{j}+q^{1 / 2}\|v\|_{0, \omega}\right) .
$$

Remark 3.2. As an example, let $v(x)=e^{\mathrm{i} k x}$ with $k \gg 1$, and $q=c^{2} k^{2} / 4$. A direct calculation leads to

$$
|v|_{s}+C \sum_{j=0}^{s-2} q^{(s-j) / 2}|v|_{j}+q^{1 / 2}\|v\|_{0, \omega}=O\left(q^{s / 2}\right)
$$

therefore, we have

$$
\left\|\pi_{M}^{q} v-v\right\|=O\left(\frac{k^{s}}{M^{s}}\right)
$$


which will converge if $\frac{k}{M}<1$. Notice that if we use the rough upper bound $(1+q)^{s / 2}$ in (3.31), the condition for convergence will be $\frac{k^{2}}{M}<1$.

A more general approximation result is stated below.

Theorem 3.2. For any $v \in \widetilde{H}_{q p}^{s}(I)$, we have that for $0 \leq r \leq s$,

$$
\left\|\pi_{M}^{q} v-v\right\|_{\widetilde{H}_{q p}^{r}(I)} \leq \lambda_{M+1}^{(r-s) / 2}\left\|\pi_{M}^{q} v-v\right\|_{\widetilde{H}_{q p}^{s}(I)} \leq \lambda_{M+1}^{(r-s) / 2}\|v\|_{\widetilde{H}_{q p}^{s}(I)} .
$$

Proof. By (3.22), we have

$$
\begin{aligned}
\| \pi_{M}^{q} v & -v \|_{\widetilde{H}_{q p}^{r}(I)}^{2}=\pi \sum_{m=M+1}^{\infty} \lambda_{m}^{r}\left|\hat{v}_{m}\right|^{2} \leq \max _{m>M}\left\{\lambda_{m}^{r-s}\right\} \sum_{m=M+1}^{\infty} \pi \lambda_{m}^{s}\left|\hat{v}_{m}\right|^{2} \\
& =\lambda_{M+1}^{r-s}\left\|\pi_{M}^{q} v-v\right\|_{\widetilde{H}_{q p}^{s}(I)}^{2} \leq \lambda_{M+1}^{r-s} \sum_{m=0}^{\infty} \pi \lambda_{m}^{s}\left|\hat{v}_{m}\right|^{2}=\lambda_{M+1}^{r-s}\|v\|_{\widetilde{H}_{q p}^{s}(I)}^{2} .
\end{aligned}
$$

This ends the proof.

As an important consequence of the above theorem, we have the following estimates in the usual Sobolev space $H_{p}^{r}(I)$ with $r \geq 1$.

Theorem 3.3. For any $v \in \widetilde{H}_{q p}^{s}(I)$,

$$
\left|\pi_{M}^{q} v-v\right|_{1} \leq \lambda_{M+1}^{(1-s) / 2}\left(1+2 \sqrt{q} \lambda_{M+1}^{-1 / 2}\right)\|v\|_{\widetilde{H}_{q p}^{s}(I)}, \quad s \geq 1
$$

and

$$
\left|\pi_{M}^{q} v-v\right|_{2} \leq \lambda_{M+1}^{1-s / 2}\left(1+2 \sqrt{q} \lambda_{M+1}^{-1}\right)\|v\|_{\widetilde{H}_{q p}^{s}(I)}, \quad s \geq 2 .
$$

In general, if $k<M$ and $1 \leq r \leq s$,

$$
\left|\pi_{M}^{q} v-v\right|_{r} \lesssim M^{r-s}\|v\|_{\widetilde{H}_{q p}^{s}(I)} .
$$

Proof. By (3.28), we have that

$$
|v|_{2 n} \leq\left\|\mathcal{A}_{q}^{n} v\right\|+C\left[\sum_{j=1}^{n-1} q^{j}\left(|v|_{2 n-2 j}+|v|_{2 n-2 j-1}\right)+q^{n}\|v\|\right],
$$

and similarly,

$$
|v|_{2 n+1} \leq\left\|\mathcal{A}_{q}^{n+1 / 2} v\right\|+C\left[\sum_{j=1}^{n-1} q^{j+1 / 2}\left(|v|_{2 n-2 j}+|v|_{2 n-2 j+1}\right)+q^{n+1 / 2}\|v\|\right] .
$$

Thus, we have that

$$
|v|_{r} \leq\|v\|_{\widetilde{H}_{q p}^{r}(I)}+C \sum_{j=0}^{r-2} q^{(r-j) / 2}|v|_{j}, \quad r \geq 2,
$$

and for $r=1,2$,

$$
\begin{aligned}
& |v|_{1} \leq\left\|\mathcal{A}_{q}^{1 / 2} v\right\|+(4 q)^{1 / 2}\|v\|_{0, \omega} \leq\|v\|_{\widetilde{H}_{q p}^{1}(I)}+2 \sqrt{q}\|v\|, \\
& |v|_{2} \leq\left\|\mathcal{A}_{q} v\right\|+2 \sqrt{q}\|v\|_{0, \omega} \leq\|v\|_{\widetilde{H}_{q p}^{2}(I)}+2 \sqrt{q}\|v\| .
\end{aligned}
$$


Hence, using Theorems 3.1 and 3.2 leads to

$$
\begin{aligned}
\left|\pi_{M}^{q} v-v\right|_{1} & \leq\left\|\pi_{M}^{q} v-v\right\|_{\widetilde{H}_{q p}^{1}(I)}+2 \sqrt{q}\left\|\pi_{M}^{q} v-v\right\| \\
& \leq \lambda_{M+1}^{(1-s) / 2}\left(1+2 \sqrt{q} \lambda_{M+1}^{-1 / 2}\right)\|v\|_{\widetilde{H}_{q p}^{s}(I)}, \quad s \geq 1
\end{aligned}
$$

and

$$
\left|\pi_{M}^{q} v-v\right|_{2} \leq \lambda_{M+1}^{1-s / 2}\left(1+2 \sqrt{q} \lambda_{M+1}^{-1}\right)\|v\|_{\widetilde{H}_{q p}^{s}(I)}, \quad s \geq 2,
$$

which yield (3.35) and (3.36).

We now prove (3.37) by induction. Assuming that (3.37) holds for $j<r$, we derive from (3.40) and Theorems 3.1 and 3.2 that for $k<M$,

$$
\begin{aligned}
\left|\pi_{M}^{q} v-v\right|_{r} & \lesssim\left\|\pi_{M}^{q} v-v\right\|_{\widetilde{H}_{q p}^{r}(I)}+\sum_{j=0}^{r-2} q^{(r-j) / 2}\left|\pi_{M}^{q} v-v\right|_{j} \\
& \lesssim\left(\lambda_{M+1}^{(r-s) / 2}+\sum_{j=0}^{r-2} q^{(r-j) / 2} M^{j-r}\right)\|v\|_{\widetilde{H}_{q p}^{s}(I)} \lesssim M^{r-s}\|v\|_{\widetilde{H}_{q p}^{s}(I)}
\end{aligned}
$$

This ends the proof.

Remark 3.3. As in Corollary 3.1, the estimates with explicit dependence on the parameter $q$ can be derived by using (3.31). Similar to the Fourier approximations, the estimate for $L^{2}$-projection in high-order Sobolev spaces is optimal.

We now estimate the projection error in $L^{\infty}$-norm.

Theorem 3.4. For any $v \in \widetilde{H}_{q p}^{s}(I)$, with $s \geq 1$,

$$
\left\|\pi_{M}^{q} v-v\right\|_{L^{\infty}} \lesssim \lambda_{M+1}^{(1-2 s) / 4}\left(1+2 \sqrt{q} \lambda_{M+1}^{-1 / 2}\right)^{1 / 2}\|v\|_{\widetilde{H}_{q p}^{s}(I)} .
$$

Proof. We recall the Sobolev embedding inequality (see, e.g., Appendix A.12 of [10])

$$
\|v\|_{L^{\infty}} \leq\left(\frac{1}{2 \pi}+2\right)^{\frac{1}{2}}\|v\|_{1}^{\frac{1}{2}}\|v\|^{\frac{1}{2}},
$$

so the desired result follows directly from Theorems 3.1 and 3.3

\section{Mathieu Approximations with $q<0$}

In this section, we extend the analysis of Mathieu approximations to the case with parameter $q<0$. Such results play an essential role in the analysis of spectral methods for elliptic and parabolic PDEs in elliptic geometries.

For clarity, let $q=-\rho$ with $\rho>0$, and let $\left\{\mathrm{ce}_{m}, \mathrm{se}_{m}\right\}$ be the Mathieu functions defined in Section 2. In view of the symmetry (2.11), we define

$$
\begin{aligned}
& \operatorname{Me}_{2 m}(\theta ; \rho)=(-1)^{\left[\frac{m}{2}\right]} \mathrm{ce}_{m}(\pi / 2-\theta ; \rho), \\
& \operatorname{Me}_{2 m+1}(\theta ; \rho)=(-1)^{\left[\frac{m}{2}\right]} \mathrm{Se}_{m+1}(\pi / 2-\theta ; \rho),
\end{aligned}
$$

for $m=0,1, \cdots$, where $[a]$ denotes the largest integer $\leq a$. Let $\left\{\lambda_{m}(\cdot)\right\}$ be the eigenvalues defined in (3.2). One verifies that the pair $\left\{\left(\lambda_{m}, \mathrm{Me}_{m}\right)\right\}$ satisfies the Sturm-Liouville problem

$$
\mathbb{A}_{\rho} \mathrm{Me}_{m}=\lambda_{m} \mathrm{Me}_{m},
$$

where the Sturm-Liouville operator is defined as

$$
\mathbb{A}_{\rho} v=\left(-\partial_{\theta}^{2}+4 \rho \sin ^{2} \theta\right) v .
$$


As the operator $\mathcal{A}_{p}$ defined in (3.4), $\mathbb{A}_{\rho}$ is also compact, strictly positive, symmetric and self-adjoint.

The following properties can be derived from the Sturm-Liouville theory or the symmetric property (2.11):

- The set of Mathieu functions $\left\{\mathrm{Me}_{m}(\theta ; \rho)\right\}$ forms a complete orthogonal system in $L^{2}(I)$, and

$$
\frac{1}{\pi} \int_{0}^{2 \pi} \mathrm{Me}_{m}(\theta ; \rho) \mathrm{Me}_{n}(\theta ; \rho) d \theta=\delta_{m n} .
$$

Moreover, they are orthogonal in $H_{p}^{1}(I)$ with the inner product

$$
\hat{a}_{\rho}(u, v)=\left(\partial_{\theta} u, \partial_{\theta} v\right)+4 \rho(u, v)_{\hat{\omega}}, \quad \text { with } \hat{\omega}=\sin ^{2} \theta,
$$

namely,

$$
\begin{aligned}
\hat{a}_{\rho}\left(\mathrm{Me}_{m}, \mathrm{Me}_{n}\right) & =\int_{0}^{2 \pi} \partial_{\theta} \mathrm{Me}_{m} \partial_{\theta} \mathrm{Me}_{n} d \theta+4 \rho \int_{0}^{2 \pi} \mathrm{Me}_{m} \mathrm{Me}_{n} \cos ^{2} \theta d \theta \\
& =\left(\mathbb{A}_{\rho} \mathrm{Me}_{m}, \mathrm{Me}_{n}\right)=\pi \lambda_{m} \delta_{m n} .
\end{aligned}
$$

- The eigenvalues $\left\{\lambda_{m}\right\}$ are real, distinct and arranged in ascending order (cf. (3.6))

$$
0<\lambda_{0}(\rho)<\lambda_{1}(\rho)<\cdots<\lambda_{m}(\rho)<\cdots,
$$

and by (3.8),

$$
m^{2}<\lambda_{m}(\rho)<m^{2}+4 \rho, \quad m=0,1, \cdots .
$$

Define the finite dimensional space

$$
\widehat{X}_{M}^{\rho}:=\operatorname{span}\left\{\mathrm{Me}_{m}: 0 \leq m \leq M\right\} .
$$

The following inequalities can be proved in the same fashion as for Lemma 3.2 .

Lemma 4.1. We have

$$
\left|\hat{a}_{\rho}(\phi, \psi)\right| \leq \lambda_{M}\|\phi\|\|\psi\| \leq\left(M^{2}+4 \rho\right)\|\phi\|\|\psi\|, \quad \forall \phi, \psi \in \widehat{X}_{M}^{\rho},
$$

and the following inverse inequality holds:

$$
\left\|\partial_{\theta}^{l} \phi\right\| \leq \lambda_{M}^{l / 2}\|\phi\|, \quad \forall \phi \in \widehat{X}_{M}^{\rho}, \quad l \geq 1 .
$$

We now consider the $L^{2}$-orthogonal projection $\hat{\pi}_{M}^{\rho}: L^{2}(I) \rightarrow \widehat{X}_{M}^{\rho}$ defined by

$$
\left(\hat{\pi}_{M}^{\rho} v-v, v_{M}\right)=0, \quad \forall v_{M} \in \widehat{X}_{M}^{\rho} .
$$

We introduce the Hilbert space $\widehat{H}_{\rho p}^{s}(I)$ with the norm $\|v\|_{\widehat{H}_{q p}^{s}(I)}$, which is defined by replacing $\mathcal{A}_{q}$ and $a_{q}(\cdot, \cdot)$ in (3.19) - (3.20) by $\mathbb{A}_{\rho}$ and $\hat{a}_{\rho}(\cdot, \cdot)$, respectively. Since the Sturm-Liouville operator $\mathbb{A}_{\rho}$ is compact, strictly positive, symmetric and selfadjoint, similar to (3.22), the norm of the space $\widehat{H}_{\rho p}^{s}(I)$ can be expressed in the frequency space

$$
\|v\|_{\widehat{H}_{\rho p}^{s}(I)}=\left(\pi \sum_{m=0}^{s} \lambda_{m}^{s}\left|\hat{v}_{m}\right|^{2}\right)^{1 / 2} \quad \text { with } \hat{v}_{m}=\frac{1}{\pi} \int_{0}^{2 \pi} v(\theta) \mathrm{Me}_{m}(\theta ; \rho) d \theta .
$$


In most applications, we may assume that the parameter $\rho$ is a finite constant independent of $M$. One verifies from a direct calculation (refer to (3.28)-(3.30) that the embedding relation $\widehat{H}_{\rho p}^{s}(I) \subset H_{p}^{s}(I)$ and

$$
\|v\|_{\widehat{H}_{\rho p}^{s}(I)} \leq C\|v\|_{s}, \quad s \geq 0
$$

where $C$ is a positive constant independent of $v$ (but depending on $\rho$ ). In the case of $\rho \gg 1$, a more precise estimate with an explicit dependence of $\rho$ can be carried out in the same fashion as in (3.31).

Following the same argument as in the proofs of Theorems 3.1 3.4, we can derive the following approximation results.

Theorem 4.1. For any $v \in \widehat{H}_{\rho p}^{s}(I)$, we have that

$$
\left\|\hat{\pi}_{M}^{\rho} v-v\right\|_{\widehat{H}_{\rho p}^{r}(I)} \leq \lambda_{M+1}^{(r-s) / 2}\|v\|_{\widehat{H}_{\rho p}^{s}(I)}, \quad 0 \leq r \leq s .
$$

In particular, for any $v \in H_{p}^{s}(I)$,

$$
\left|\hat{\pi}_{M}^{\rho} v-v\right|_{r} \lesssim M^{r-s}\|v\|_{s}, \quad 0 \leq r \leq s
$$

and

$$
\left\|\hat{\pi}_{M^{\rho}}^{\rho} v-v\right\|_{L^{\infty}} \lesssim M^{1 / 2-s}\|v\|_{s}, \quad s \geq 1
$$

In the preceding part, we presented Mathieu approximation results in Sobolev spaces, which are the main ingredients for the analysis of spectral approximation to PDEs in elliptical geometries, as illustrated in the succeeding two sections.

\section{Application to the modified Helmholtz equation}

We first consider the modified Helmholtz equation in an ellipse:

$$
-\Delta U+\beta U=F, \quad \text { in } \Omega ; \quad U(x, y)=0, \quad \text { on } \quad \partial \Omega,
$$

where $\beta \geq 0, F$ is a given function and

$$
\Omega=\left\{(x, y): \frac{x^{2}}{c^{2} \cosh ^{2} 1}+\frac{y^{2}}{c^{2} \sinh ^{2} 1}<1\right\}
$$

with $c$ being the semi-focal distance.

Using the elliptic transform (2.1) and setting $u(\mu, \theta)=U(x, y)$, we have that

$$
\nabla U(x, y)=\frac{1}{h} \frac{\partial u}{\partial \mu} \overrightarrow{\mathbf{e}}_{\mu}+\frac{1}{h} \frac{\partial u}{\partial \theta} \overrightarrow{\mathbf{e}}_{\theta}:=\frac{1}{h} \tilde{\nabla} u
$$

and

$$
\Delta U(x, y)=\frac{1}{h^{2}}\left(\frac{\partial^{2} u}{\partial \mu^{2}}+\frac{\partial^{2} u}{\partial \theta^{2}}\right):=\frac{1}{h^{2}} \tilde{\Delta} u
$$

where

$$
h=h(\mu, \theta)=c\left(\cosh ^{2} \mu-\cos ^{2} \theta\right)^{1 / 2}=\frac{c}{\sqrt{2}}(\cosh 2 \mu-\cos 2 \theta)^{1 / 2},
$$

and the Jacobian from $(x, y)$ to $(\mu, \theta)$ is $h^{2}$, i.e., $d x d y=h^{2} d \mu d \theta$. Hence, under the elliptic coordinates, equation (5.1) takes the form

$$
\begin{aligned}
& -\frac{1}{h^{2}} \tilde{\Delta} u+\beta u=f, \quad(\mu, \theta) \in \mathcal{Q}:=(0,1) \times[0,2 \pi), \\
& u(1, \theta)=0, \quad \theta \in[0,2 \pi) ; \quad u \text { is } 2 \pi \text {-periodic in } \theta,
\end{aligned}
$$


where $f(\mu, \theta)=F(x, y)$.

We note that curves of constant $\mu$ are all ellipses and the curves of constant $\theta$ are hyperbolas with foci $x= \pm c$ along the $x$-axis. The transform (2.1) is singular at $\mu=0$ when the ellipse is reduced to the line segment $y=0$ and $|x| \leq c 1$ Therefore, additional condition(s) should be imposed for the solution of (5.6) to be consistent with that of (5.1). We now derive the necessary condition(s) through weak formulations.

The weak formulation of (5.1) is to find $U \in H_{0}^{1}(\Omega)$ such that

$$
\int_{\Omega} \nabla U \nabla V d x d y+\beta \int_{\Omega} U V d x d y=\int_{\Omega} F V d x d y, \quad \forall V \in H_{0}^{1}(\Omega) .
$$

By the Lax-Milgram Lemma, if $\beta \geq 0$, this problem admits a unique solution $U \in H_{0}^{1}(\Omega)$, provided that $F \in L^{2}(\Omega)$.

Let us define

$$
X:=\left\{u \in L_{h^{2}}^{2}(\mathcal{Q}): \tilde{\nabla} u \in L^{2}(\mathcal{Q}), u(1, \theta)=0, u(\mu, \theta+2 \pi)=u(\mu, \theta)\right\} .
$$

Using (5.3), we find that, under the elliptic coordinates, the weak formulation (5.7) becomes: find $u \in X$ such that

$$
\mathcal{B}(u, v):=\int_{\mathcal{Q}} \tilde{\nabla} u \tilde{\nabla} v d \mu d \theta+\beta \int_{\mathcal{Q}} u v h^{2} d \mu d \theta=\int_{\mathcal{Q}} f v h^{2} d \mu d \theta, \quad \forall v \in X .
$$

On the other hand, in order for (5.6) to be consistent with (5.9), the condition

$$
\frac{\partial u}{\partial \mu}(0, \theta)=0, \quad \forall \theta \in[0,2 \pi)
$$

should be supplemented to (5.6).

5.1. Dimension reduction. We now expand the solution in a series of Mathieu functions in $\theta$-direction:

$$
\left(u, h^{2} f\right)=\sum_{m=0}^{\infty}\left(\hat{u}_{m}, \hat{f}_{m}\right) \mathrm{Me}_{m}(\theta ; \rho)
$$

with $\rho=c^{2} \beta / 4$. Since the Mathieu function $\mathrm{Me}_{m}$ satisfies the Mathieu equation (4.2), substituting the expansion (5.11) into (5.6) and (5.10) leads to a sequence of one-dimensional problems in the $\mu$-direction:

$$
\begin{aligned}
& -\hat{u}_{m}^{\prime \prime}+\left(\lambda_{m}+4 \rho \cosh ^{2} \mu\right) \hat{u}_{m}=\hat{f}_{m}, \quad \forall \mu \in \Lambda=(0,1), \\
& \hat{u}_{m}^{\prime}(0)=\hat{u}_{m}(1)=0, \quad m=0,1, \cdots .
\end{aligned}
$$

Its weak formulation is:

$$
\begin{aligned}
& \text { Find } \hat{u}_{m} \in{ }_{0} H^{1}(\Lambda)=\left\{\phi: \phi \in H^{1}(\Lambda), \phi(1)=0\right\} \text { such that } \\
& \mathcal{B}_{m}\left(\hat{u}_{m}, \hat{v}_{m}\right)=\left(\hat{f}_{m}, \hat{v}_{m}\right), \quad \forall \hat{v}_{m} \in{ }_{0} H^{1}(\Lambda), \quad m=0,1, \cdots,
\end{aligned}
$$

where

$$
\mathcal{B}_{m}\left(\hat{u}_{m}, \hat{v}_{m}\right)=\left(\hat{u}_{m}^{\prime}, \hat{v}_{m}^{\prime}\right)+\lambda_{m}\left(\hat{u}_{m}, \hat{v}_{m}\right)+4 \rho\left(\hat{u}_{m}, \hat{v}_{m}\right)_{\chi}
$$

with the weight function $\chi=\cosh ^{2} \mu$. For each $m \geq 0$, the problem is uniquely solvable thanks to the fact $\lambda_{m}, \rho>0$ and the Lax-Milgram lemma.

\footnotetext{
${ }^{1}$ The singularity here is different from that of the polar coordinates, because $\mu=0$ corresponds to the whole interval $[-c, c]$ rather than a single point.
} 
Moreover, we have the following a priori estimate whose proof is given in Appendix C.

Lemma 5.1. For each $m \geq 0$, if $\hat{f}_{m} \in L^{2}(\Lambda)$, then we have

$$
\left\|\hat{u}_{m}\right\|_{2}^{2}+\lambda_{m}\left\|\hat{u}_{m}\right\|_{1}^{2}+\lambda_{m}^{2}\left\|\hat{u}_{m}\right\|^{2} \lesssim\left\|\hat{f}_{m}\right\|^{2} .
$$

5.2. Mixed Mathieu-Legendre spectral-Galerkin methods. We now describe our spectral approximation to (5.6) with (5.10). Given a cutoff integer $M>0$, we define the Mathieu spectral approximation to the solution of (5.9) by

$$
u_{M N}(\mu, \theta)=\sum_{m=0}^{M} \hat{u}_{m}^{N}(\mu) \mathrm{Me}_{m}(\theta ; \rho),
$$

where $\left\{\hat{u}_{m}^{N}\right\}_{m=0}^{M}$ are the solutions of the following Legendre spectral-Galerkin approximation to (5.13):

$$
\begin{aligned}
& \text { Find } \hat{u}_{m}^{N} \in{ }_{0} P_{N}:=\left\{\phi \in P_{N}: \phi(1)=0\right\} \text { such that } \\
& \mathcal{B}_{m}\left(\hat{u}_{m}^{N}, \hat{v}_{m}^{N}\right)=\left(\hat{f}_{m}, \hat{v}_{m}^{N}\right), \quad \forall \hat{v}_{m}^{N} \in{ }_{0} P_{N}, \quad m=0,1, \cdots, M,
\end{aligned}
$$

where the bilinear form $\mathcal{B}_{m}(\cdot, \cdot)$ is defined in (5.14).

5.3. Error estimations. We first recall some relevant approximation results by Legendre polynomials (with a change of variable from $(-1,1)$ to $\Lambda:=(0,1)$ ). Consider the orthogonal projection ${ }_{0} \Pi_{N}^{1}:{ }_{0} H^{1}(\Lambda) \rightarrow{ }_{0} P_{N}$ such that

$$
\left(\partial_{\mu}\left({ }_{0} \Pi_{N}^{1} v-v\right), \partial_{\mu} v_{N}\right)+\left({ }_{0} \Pi_{N}^{1} v-v, v_{N}\right)=0, \quad \forall v_{N} \in{ }_{0} P_{N} .
$$

As in [16, we introduce the weighted space to describe the approximation errors:

$$
B^{r}(\Lambda):=\left\{v \in L^{2}(\Lambda):\left(\mu-\mu^{2}\right)^{\frac{k-1}{2}} \partial_{\mu}^{k} v \in L^{2}(\Lambda), \quad 1 \leq k \leq r\right\}
$$

with the norm and semi-norm

$\|v\|_{B^{r}(\Lambda)}=\left(\|v\|^{2}+\sum_{k=1}^{r}\left\|\left(\mu-\mu^{2}\right)^{\frac{k-1}{2}} \partial_{\mu}^{k} v\right\|^{2}\right)^{\frac{1}{2}}, \quad|v|_{B^{r}(\Lambda)}=\left\|\left(\mu-\mu^{2}\right)^{\frac{r-1}{2}} \partial_{\mu}^{r} v\right\|, r \geq 1$.

In particular, we have $B^{0}(\Lambda)=L^{2}(\Lambda)$.

The following lemma can be derived from the existing Legendre polynomial approximation; see, e.g., [13, 16].

Lemma 5.2. For any $v \in{ }_{0} H^{1}(\Lambda) \cap B^{r}(\Lambda)$,

$$
\left\|_{0} \Pi_{N}^{1} v-v\right\|_{\sigma} \lesssim N^{\sigma-r}\left\|\left(\mu-\mu^{2}\right)^{(r-1) / 2} \partial_{\mu}^{r} v\right\|, \quad 0 \leq \sigma \leq 1 \leq r .
$$

We first estimate the error between $\hat{u}_{m}$ (the solution of (5.13)) and $\hat{u}_{m}^{N}$ (the solution of (5.17)). For notational convenience, denote $\hat{e}_{m}=\hat{u}_{m}-\hat{u}_{m}^{N}$ and $\hat{e}_{m}^{N}=$ ${ }_{0} \Pi_{N}^{1} \hat{u}_{m}-\hat{u}_{m}^{N}$. Clearly, we have that ${ }_{0} \Pi_{N}^{1} \hat{u}_{m}-\hat{u}_{m}=\hat{e}_{m}^{N}-\hat{e}_{m}:=\tilde{e}_{m}^{N}$.

Lemma 5.3. For each $m \geq 0$, if $\hat{u}_{m} \in{ }_{0} H^{1}(\Lambda) \cap B^{r}(\Lambda)$ with $r \geq 1$, then we have

$$
\sqrt{\mathcal{B}_{m}\left(\hat{u}_{m}-\hat{u}_{m}^{N}, \hat{u}_{m}-\hat{u}_{m}^{N}\right)} \lesssim\left(\lambda_{m}^{1 / 2}+N\right) N^{-r}\left|\hat{u}_{m}\right|_{B^{r}(\Lambda)}
$$

and

$$
\left\|\hat{u}_{m}-\hat{u}_{m}^{N}\right\| \lesssim\left(\lambda_{m}+N^{2}\right) N^{-(2+r)}\left|\hat{u}_{m}\right|_{B^{r}(\Lambda)} .
$$


Proof. By (5.13) and (5.17), we have that for any $\hat{v}_{m}^{N} \in{ }_{0} P_{N}$,

$$
\mathcal{B}_{m}\left(\hat{e}_{m}, \hat{v}_{m}^{N}\right)=0 \Rightarrow \mathcal{B}_{m}\left(\hat{e}_{m}^{N}, \hat{v}_{m}^{N}\right)=\mathcal{B}_{m}\left(\tilde{e}_{m}^{N}, \hat{v}_{m}^{N}\right) .
$$

Taking $\hat{v}_{m}^{N}=\hat{e}_{m}^{N}$ in the above identity, we derive from (5.14), (5.18) and the CauchySchwarz inequality that

$$
\begin{aligned}
\mathcal{B}_{m}\left(\hat{e}_{m}^{N}, \hat{e}_{m}^{N}\right)=\left(\lambda_{m}-1\right)\left(\tilde{e}_{m}^{N}, \hat{e}_{m}^{N}\right)+4 \rho\left(\tilde{e}_{m}^{N}, \hat{e}_{m}^{N}\right)_{\chi} \\
\quad \leq \lambda_{m}\left\|\tilde{e}_{m}^{N}\right\|\left\|\hat{e}_{m}^{N}\right\|+\left\|\tilde{e}_{m}^{N}\right\|\left\|\hat{e}_{m}^{N}\right\|+4 \rho\left\|\tilde{e}_{m}^{N}\right\|_{0, \chi}\left\|\hat{e}_{m}^{N}\right\|_{0, \chi} \\
\leq \frac{\lambda_{m}}{2}\left\|\hat{e}_{m}^{N}\right\|^{2}+\frac{\lambda_{m}}{2}\left\|\tilde{e}_{m}^{N}\right\|^{2}+\frac{1}{2}\left|\hat{e}_{m}^{N}\right|_{1}^{2}+2\left\|\tilde{e}_{m}^{N}\right\|^{2}+2 \rho\left\|\hat{e}_{m}^{N}\right\|_{0, \chi}^{2}+2 \rho\left\|\tilde{e}_{m}^{N}\right\|_{0, \chi}^{2} \\
\quad=\frac{1}{2} \mathcal{B}_{m}\left(\hat{e}_{m}^{N}, \hat{e}_{m}^{N}\right)+\frac{\lambda_{m}}{2}\left\|\tilde{e}_{m}^{N}\right\|^{2}+2 \rho\left\|\tilde{e}_{m}^{N}\right\|_{0, \chi}^{2}+2\left\|\tilde{e}_{m}^{N}\right\|^{2},
\end{aligned}
$$

where we used the embedding inequality (B.1) for the following derivation:

$$
\left\|\hat{e}_{m}^{N}\right\| \leq 2\left|\hat{e}_{m}^{N}\right|_{1} \quad \Rightarrow \quad\left\|\tilde{e}_{m}^{N}\right\|\left\|\hat{e}_{m}^{N}\right\| \leq 2\left\|\tilde{e}_{m}^{N}\right\|\left|\hat{e}_{m}^{N}\right|_{1} \leq \frac{1}{2}\left|\hat{e}_{m}^{N}\right|_{1}^{2}+2\left\|\tilde{e}_{m}^{N}\right\|^{2} .
$$

Therefore, we derive from Lemma 5.2 that for $r \geq 1$,

$$
\begin{aligned}
\mathcal{B}_{m}\left(\hat{e}_{m}^{N}, \hat{e}_{m}^{N}\right) & \leq \lambda_{m}\left\|\tilde{e}_{m}^{N}\right\|^{2}+4\left\|\tilde{e}_{m}^{N}\right\|^{2}+4 \rho\left\|\tilde{e}_{m}^{N}\right\|_{0, \chi}^{2} \\
& \lesssim\left(\lambda_{m}+1\right) N^{-2 r}\left\|\left(\mu-\mu^{2}\right)^{(r-1) / 2} \partial_{\mu}^{r} \hat{u}_{m}\right\|^{2} .
\end{aligned}
$$

Since by the triangle inequality,

$$
\mathcal{B}_{m}\left(\hat{e}_{m}, \hat{e}_{m}\right) \leq \mathcal{B}_{m}\left(\hat{e}_{m}^{N}, \hat{e}_{m}^{N}\right)+\mathcal{B}_{m}\left(\tilde{e}_{m}^{N}, \tilde{e}_{m}^{N}\right)
$$

we obtain the desired result by using (5.25) and Lemma 5.2

We now estimate (5.22) by a duality argument. Given $g \in L^{2}(\Lambda)$, we consider the dual problem of (5.13): Find $w \in{ }_{0} H^{1}(\Lambda)$ such that

$$
\mathcal{B}_{m}(w, v)=(g, v), \quad \forall v \in{ }_{0} H^{1}(\Lambda) .
$$

By an argument similar to that for Lemma 5.1, this problem admits a unique solution with the regularity

$$
\|w\|_{2}^{2}+\lambda_{m}\|w\|_{1}^{2}+\lambda_{m}^{2}\|w\|^{2} \lesssim\|g\|^{2} .
$$

Taking $v=\hat{e}_{m}$ in (5.27), we deduce from (5.23), the Cauchy-Schwarz inequality and (5.21) that

$$
\begin{aligned}
\left|\left(g, \hat{e}_{m}\right)\right|^{2} & =\left|\mathcal{B}_{m}\left(w, \hat{e}_{m}\right)\right|^{2}=\left|\mathcal{B}_{m}\left(w-{ }_{0} \Pi_{N}^{1} w, \hat{e}_{m}\right)\right|^{2} \\
& \leq \mathcal{B}_{m}\left(w-{ }_{0} \Pi_{N}^{1} w, w-{ }_{0} \Pi_{N}^{1} w\right) \mathcal{B}_{m}\left(\hat{e}_{m}, \hat{e}_{m}\right) \\
& \lesssim\left(\lambda_{m}^{1 / 2}+N\right)^{4} N^{-(4+2 r)}\left\|\left(\mu-\mu^{2}\right)^{1 / 2} w^{\prime \prime}\right\|^{2}\left|\hat{u}_{m}\right|_{B^{r}(\Lambda)}^{2} \\
& \lesssim\left(\lambda_{m}^{1 / 2}+N\right)^{4} N^{-(4+2 r)}\|g\|^{2}\left|\hat{u}_{m}\right|_{B^{r}(\Lambda)}^{2} .
\end{aligned}
$$

Consequently,

$$
\left\|\hat{e}_{m}\right\|=\sup _{g \in L^{2}, g \neq 0} \frac{\left|\left(g, \hat{e}_{m}\right)\right|}{\|g\|} \lesssim\left(\lambda_{m}^{1 / 2}+N\right)^{2} N^{-(2+r)}\left|\hat{u}_{m}\right|_{B^{r}(\Lambda)} .
$$

This ends the proof. 
We now estimate the global errors between $u$ (the solution of (5.9)) and $u_{M N}$ (the numerical solution defined in (5.16)-(5.17)). For this purpose, we introduce the Sobolev space

$$
H_{p}^{r, s}(\mathcal{Q}):=L^{2}\left(I ; B^{r}(\Lambda)\right) \cap H_{p}^{s-1}\left(I ;{ }_{0} H^{1}(\Lambda)\right) \cap H_{p}^{s}\left(I ; L^{2}(\Lambda)\right), \quad r, s \geq 1,
$$

with the norm

$$
\|u\|_{H_{p}^{r, s}(\mathcal{Q})}=\left(\pi \sum_{m=0}^{\infty}\left[\left|\hat{u}_{m}\right|_{B^{r}(\Lambda)}^{2}+\lambda_{m}^{s-1}\left\|\hat{u}_{m}\right\|_{H^{1}(\Lambda)}^{2}+\lambda_{m}^{s}\left\|\hat{u}_{m}\right\|_{L^{2}(\Lambda)}^{2}\right]\right)^{\frac{1}{2}},
$$

where $\left\{\hat{u}_{m}\right\}$ are the coefficients of the Mathieu expansion of $u$.

With the above preparations, we now estimate the global error.

Theorem 5.1. Let $u$ be the solution of (5.9) and $u_{M N}$ its approximation given by (5.16) -(5.17). Then, for $u \in H_{p}^{r, s}(\mathcal{Q})$ with $r, s \geq 1$, we have

$$
\left\|\tilde{\nabla}\left(u-u_{M N}\right)\right\|_{L^{2}(\mathcal{Q})} \lesssim\left(M^{1-s}+(M+N) N^{-r}\right)\|u\|_{H_{p}^{r, s}(\mathcal{Q})}
$$

and

$$
\left\|u-u_{M N}\right\|_{L^{2}(\mathcal{Q})} \lesssim\left(M^{-s}+\left(M^{2}+N^{2}\right) N^{-2-r}\right)\|u\|_{H_{p}^{r, s}(\mathcal{Q})} .
$$

Proof. Recall that $\hat{e}_{m}=\hat{u}_{m}-\hat{u}_{m}^{N}$. Hence, by (5.11) and (5.16),

$$
u-u_{M N}=\sum_{m=0}^{M} \hat{e}_{m} \mathrm{Me}_{m}+\sum_{m=M+1}^{\infty} \hat{u}_{m} \mathrm{Me}_{m} .
$$

Using (5.9), (4.2)-(4.6) and a direct calculation leads to

$$
\begin{aligned}
\mathcal{B}(u & \left.-u_{M N}, u-u_{M N}\right)=\left\|\tilde{\nabla}\left(u-u_{M N}\right)\right\|_{L^{2}(\mathcal{Q})}^{2}+\beta\left\|u-u_{M N}\right\|_{L_{h^{2}}^{2}(\mathcal{Q})}^{2} \\
= & \sum_{m=0}^{M}\left[\pi\left\|\hat{e}_{m}^{\prime}\right\|_{L^{2}(\Lambda)}^{2}+4 \rho \pi\left\|\hat{e}_{m}\right\|_{L_{\chi}^{2}(\Lambda)}^{2}+\left\|\hat{e}_{m}\right\|_{L^{2}(\Lambda)}^{2} \hat{a}_{\rho}\left(\mathrm{Me}_{m}, \mathrm{Me}_{m}\right)\right] \\
& +\sum_{m=M+1}^{\infty}\left[\pi\left\|\hat{u}_{m}^{\prime}\right\|_{L^{2}(\Lambda)}^{2}+4 \rho \pi\left\|\hat{u}_{m}\right\|_{L_{\chi}^{2}(\Lambda)}^{2}+\left\|\hat{u}_{m}\right\|_{L^{2}(\Lambda)}^{2} \hat{a}_{\rho}\left(\mathrm{Me}_{m}, \mathrm{Me}_{m}\right)\right] \\
= & \pi \sum_{m=0}^{M} \mathcal{B}_{m}\left(\hat{e}_{m}, \hat{e}_{m}\right)+\pi \sum_{m=M+1}^{\infty} \mathcal{B}_{m}\left(\hat{u}_{m}, \hat{u}_{m}\right),
\end{aligned}
$$

where $h^{2}$ is defined in (5.5), and the weight function $\chi=\cosh ^{2} \mu$. By Lemma 5.3 and (5.14),

$$
\sum_{m=0}^{M} \mathcal{B}_{m}\left(\hat{e}_{m}, \hat{e}_{m}\right) \lesssim\left(\lambda_{M}^{1 / 2}+N\right)^{2} N^{-2 r} \sum_{m=0}^{M}\left|\hat{u}_{m}\right|_{B^{r}(\Lambda)}^{2} \leq(M+N)^{2} N^{-2 r}\|u\|_{H_{p}^{r, s}(\mathcal{Q})}^{2} .
$$

On the other hand, we have from (4.8) and (5.14) that

$$
\begin{aligned}
\sum_{m=M+1}^{\infty} \mathcal{B}_{m}\left(\hat{u}_{m}, \hat{u}_{m}\right) \leq \lambda_{M+1}^{1-s} \sum_{m=M+1}^{\infty} \lambda_{m}^{s-1} \mathcal{B}_{m}\left(\hat{u}_{m}, \hat{u}_{m}\right) \\
\lesssim M^{2-2 s} \sum_{m=M+1}^{\infty}\left(\lambda_{m}^{s-1}\left\|\hat{u}_{m}\right\|_{H^{1}(\Lambda)}^{2}+\lambda_{m}^{s}\left\|\hat{u}_{m}\right\|_{L^{2}(\Lambda)}^{2}\right)
\end{aligned}
$$


Therefore, we deduce from the above estimates and (5.31) -(5.32) that

$\left\|\tilde{\nabla}\left(u-u_{M N}\right)\right\|_{L^{2}(\mathcal{Q})}+\sqrt{\beta}\left\|u-u_{M N}\right\|_{L_{h^{2}}^{2}(\mathcal{Q})} \lesssim\left(M^{1-s}+(M+N) N^{-r}\right)\|u\|_{H_{p}^{r, s}(\mathcal{Q})}$.

This yields (5.33).

We now prove (5.34). By (5.35), the orthogonality (4.4) and (5.22),

$$
\begin{aligned}
\| u & -u_{M N}\left\|_{L^{2}(\mathcal{Q})}^{2}=\pi \sum_{m=0}^{M}\right\| \hat{e}_{m}\left\|_{L^{2}(\Lambda)}^{2}+\pi \sum_{m=M+1}^{\infty}\right\| \hat{u}_{m} \|_{L^{2}(\Lambda)}^{2} \\
& \lesssim\left(M^{2}+N^{2}\right)^{2} N^{-2(2+r)} \sum_{m=0}^{M}\left|\hat{u}_{m}\right|_{B^{r}(\Lambda)}^{2}+\lambda_{m}^{-2 s} \sum_{m=M+1}^{\infty} \lambda_{m}^{2 s}\left\|\hat{u}_{m}\right\|_{L^{2}(\Lambda)}^{2} \\
& \lesssim\left(M^{-2 s}+\left(M^{2}+N^{2}\right)^{2} N^{-2(2+r)}\right)\|u\|_{H_{p}^{r, s}(\mathcal{Q})}^{2} .
\end{aligned}
$$

This ends the proof.

We now present some numerical results. As a first example, we consider (5.9) with $\beta=c=1$ and the exact solution

$$
U(x, y)=\cos (x y)=v(\mu, \theta)=\cos (\sinh (2 \mu) \sin (2 \theta) / 4) .
$$

For this analytical solution, Theorem 5.1 holds for all $r, s \geq 0$ hence an exponential convergence is expected. We plot the $L^{2}$-errors against various $M=N$ on the left side of Figure 5.1. An exponential convergence rate of order $O\left(e^{-c N}\right)$ is clearly observed.

For the second example, we consider (5.9) with the exact solution $v(\mu, \theta)=$ $\mu^{\gamma} \sin (2 \theta)$. It can be easily checked that for $\gamma \geq \frac{3}{2}$ but noninteger, we have $v \in$ $H_{p}^{2 \gamma-\varepsilon, s}(\mathcal{Q})$ for any $\varepsilon, s>0$. Hence, Theorem 5.1 indicates an $L^{2}$ convergence rate of $N^{-2 \gamma+\varepsilon}$ with $M=N$ for any $\varepsilon>0$. We plot the $L^{2}$-errors vs. various $M=N$ in $\log$ - $\log$ scale for $\gamma=2.5,2.8,3.5$ on the right side of Figure 5.1. We observe algebraic decays with slopes of roughly $2-2 \gamma$. We note that the difference between the observed rates and the rates predicted in Theorem 5.1 can be attributed to the fact that the forcing function $f$ is replaced by its Mathieu-Legendre interpolation in the computation and that this interpolation error is not accounted for in Theorem 5.1 .
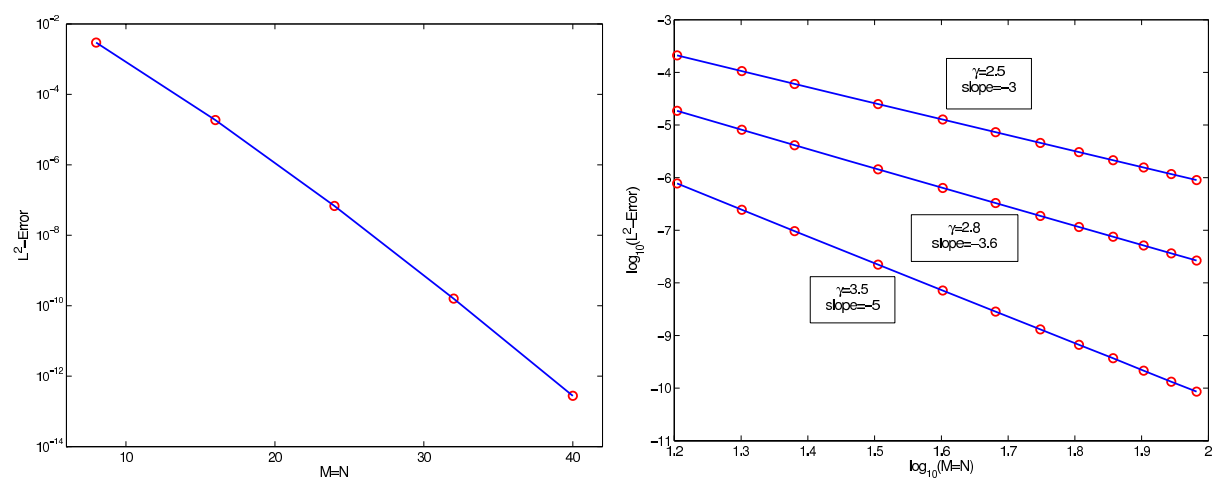

Figure 5.1. Left: $L^{2}$-errors in semi-log scale for the first example.

Right: $L^{2}$-errors in log-log scale for the second example. 


\section{Application to the Helmholtz equation}

Given $r>0$, we denote

$$
D_{r}=\left\{(x, y): \frac{x^{2}}{c^{2} \cosh ^{2} r}+\frac{y^{2}}{c^{2} \sinh ^{2} r}<1\right\}
$$

where $c>0$ is the semi-focal distance. For $b>a>0$, we denote $\Omega_{a, b}=D_{b} \backslash \bar{D}_{a}$, i.e., the open domain between the two co-focal ellipses. We consider the following Helmholtz equation

$$
-\Delta V-k^{2} V=G, \quad \text { in } \Omega_{a, b} ;\left.\quad V\right|_{\partial D_{a}}=0,
$$

together with a Robin boundary condition at $\partial D_{b}$ expressed in elliptic coordinates (see [14]):

$$
\frac{\partial v}{\partial \mu}-\left(\mathrm{i} c k \sinh \mu-\frac{1}{2} \tanh \mu\right) v=0, \quad \text { on } \partial D_{b},
$$

where $v(\mu, \theta)=V(x, y)$. We note that this boundary condition is a first-order approximation to the exact Dirichlet-to-Neumann boundary condition (cf. 14]). Without loss of generality, we consider only the homogeneous boundary conditions since nonhomogeneous boundary conditions can be easily handled by boundary lifting.

Under the elliptic coordinates (2.1), the equation (6.2) $-(6.3)$ becomes

$$
\begin{aligned}
& -\frac{1}{h^{2}}\left(\frac{\partial^{2} v}{\partial \mu^{2}}+\frac{\partial^{2} v}{\partial \theta^{2}}\right)-k^{2} v=g, \quad \forall(\mu, \theta) \in(a, b) \times[0,2 \pi), \\
& v(a, \theta)=0, \quad\left(\frac{\partial v}{\partial \mu}-\left(\mathrm{i} c k \sinh \mu-\frac{1}{2} \tanh \mu\right) v\right)(b, \theta)=0, \quad \forall \theta \in[0,2 \pi),
\end{aligned}
$$

$v$ is $2 \pi$-periodic in $\theta$,

where $g(\mu, \theta)=G(x, y)$.

6.1. Dimension reduction. We expand the solution and given data in a series of Mathieu functions in the $\theta$-direction

$$
\left(v, h^{2} g\right)=\sum_{m=0}^{\infty}\left(\hat{v}_{m}, \hat{g}_{m}\right) \mathrm{me}_{m}(\theta ; q)
$$

with $q=c^{2} k^{2} / 4$. For simplicity, hereafter, we denote $\hat{k}=c k$. The equation (6.4) is reduced to

$$
\begin{gathered}
-\hat{v}_{m}^{\prime \prime}+\left(\lambda_{m}-\hat{k}^{2} \cosh ^{2} \mu\right) \hat{v}_{m}=\hat{g}_{m}, \quad \forall \mu \in \Lambda=(a, b), \\
\hat{v}_{m}(a)=0, \quad \hat{v}_{m}^{\prime}(b)-\mathcal{T}_{\hat{k}, b} \hat{v}_{m}(b)=0, \quad m=0,1, \cdots,
\end{gathered}
$$

where

$$
\mathcal{T}_{\hat{k}, b}:=\mathrm{i} \hat{k} \sinh b-\frac{1}{2} \tanh b .
$$

We note that the functions $(v, g)$ and $\left(\hat{v}_{m}, \hat{g}_{m}\right)$ are complex-valued. With a slight abuse of notation, we shall still use $H^{s}$ and $L^{2}$, etc. to denote spaces of complexvalued functions. 
Let $(u, v):=\int_{\Lambda} u \bar{v} d \mu$. Then, a weak formulation of (6.6) is: Find $\hat{v}_{m} \in{ }^{0} H^{1}(\Lambda):=$ $\left\{v \in H^{1}(\Lambda): v(a)=0\right\}$ such that

$$
\begin{gathered}
\mathbb{B}_{m}\left(\hat{v}_{m}, \hat{w}_{m}\right):=\left(\hat{v}_{m}^{\prime}, \hat{w}_{m}^{\prime}\right)+\lambda_{m}\left(\hat{v}_{m}, \hat{w}_{m}\right)-\hat{k}^{2}\left(\hat{v}_{m}, \hat{w}_{m}\right)_{\chi} \\
-\mathcal{T}_{\hat{k}, b} \hat{v}_{m}(b) \overline{\hat{w}_{m}(b)}=\left(\hat{g}_{m}, \hat{w}_{m}\right), \quad \forall \hat{w}_{m} \in{ }^{0} H^{1}(\Lambda),
\end{gathered}
$$

where $\chi=\cosh ^{2} \mu$.

Theorem 6.1. Given $m \geq 0$ and $\hat{g}_{m} \in L^{2}(\Lambda)$, problem (6.8) admits a unique solution $\hat{v}_{m} \in H^{2}(\Lambda) \cap{ }^{0} H^{1}(\Lambda)$.

Proof. Observe that $\operatorname{Im}\left(\mathcal{T}_{\hat{k}, b}\right)=\hat{k} \sinh b>0$, so we deduce from the Fredholm alternative theorem (see, for instance, 22 , 31, 34]) that if $\hat{g}_{m} \in L^{2}(\Lambda)$, the problem (6.8) has a unique solution $\hat{v}_{m} \in{ }^{0} H^{1}(\Lambda)$. Then by a standard regularity argument, we have

$$
\left\|\hat{v}_{m}\right\|_{2} \lesssim c(a, b, \hat{k})\left\|\hat{g}_{m}\right\|
$$

where $c(a, b, \hat{k})$ is a positive constant depending on $a, b$ and $\hat{k}$.

We now derive a sharp a prior estimate with explicit dependence on $\hat{k}$.

Lemma 6.1. Given $m \geq 0$ and $\hat{g}_{m} \in L^{2}(\Lambda) \cap L_{\hat{\chi}^{-1}}^{2}(\Lambda)$, we have

$$
\left\|\hat{v}_{m}^{\prime}\right\|+\frac{\hat{k}}{2}\left\|\hat{v}_{m}\right\|_{0, \hat{\chi}} \leq C_{a, b, \hat{k}}\left\|\hat{g}_{m}\right\|_{0, \hat{\chi}^{-1}}+(b-a+1)\left\|\hat{g}_{m}\right\|
$$

where

$$
\begin{aligned}
& \hat{\chi}(\mu)=(\mu-a+1) \sinh (2 \mu), \\
& C_{a, b, \hat{k}}=\sqrt{2}(b-a+1)\left[\frac{\cosh (2 b)}{\sinh b}+\frac{\tanh ^{2} b}{4 \hat{k}^{2} \sinh b}\right]+\frac{1}{\hat{k}} .
\end{aligned}
$$

Proof. In order to derive the desired a priori estimate, we take three different test functions in (6.8), namely, $\hat{v}_{m},(\mu-a) \hat{v}_{m}^{\prime}$ and $\hat{v}_{m}^{\prime}$. The following weight functions will be used in the sequel:

$$
\begin{gathered}
\chi(\mu)=\cosh ^{2} \mu, \tilde{\chi}(\mu)=(\mu-a) \sinh (2 \mu), \\
\bar{\chi}(\mu)=\sinh (2 \mu), \hat{\chi}(\mu)=(\mu-a+1) \sinh (2 \mu) .
\end{gathered}
$$

Step 1. Taking $\hat{w}_{m}=\hat{v}_{m}$ in (6.8) leads to

$$
\left\|\hat{v}_{m}^{\prime}\right\|^{2}+\lambda_{m}\left\|\hat{v}_{m}\right\|^{2}-\hat{k}^{2}\left\|\hat{v}_{m}\right\|_{0, \chi}^{2}-\mathcal{T}_{\hat{k}, b}\left|\hat{v}_{m}(b)\right|^{2}=\int_{a}^{b} \hat{g}_{m} \overline{\hat{v}_{m}} d \mu
$$

whose real and imaginary parts read

$$
\left\|\hat{v}_{m}^{\prime}\right\|^{2}+\lambda_{m}\left\|\hat{v}_{m}\right\|^{2}+\frac{1}{2} \tanh b\left|\hat{v}_{m}(b)\right|^{2}=\hat{k}^{2}\left\|\hat{v}_{m}\right\|_{0, \chi}^{2}+\operatorname{Re}\left(\hat{g}_{m}, \hat{v}_{m}\right)
$$

and

$$
-\mathrm{i} \hat{k} \sinh b\left|\hat{v}_{m}(b)\right|^{2}=\operatorname{Im}\left(\hat{g}_{m}, \hat{v}_{m}\right)
$$

Theorem 6.1 asserts that the solution $\hat{v}_{m}$ of (6.8) also solves the original problem (6.6). Therefore, we have that for any $\hat{w}_{m} \in H^{1}(\Lambda)$,

$$
\int_{a}^{b}\left(-\hat{v}_{m}^{\prime \prime}+\left(\lambda_{m}-\hat{k}^{2} \cosh ^{2} \mu\right) \hat{v}_{m}\right) \overline{\hat{w}_{m}} d \mu=\int_{a}^{b} \hat{g}_{m} \overline{\hat{w}_{m}} d \mu .
$$


Step 2. In order to bound the term $\hat{k}^{2}\left\|\hat{v}_{m}\right\|_{0, \chi}^{2}$ in (6.12), we take $\hat{w}_{m}=2(\mu-a) \hat{v}_{m}^{\prime}$ in (6.14) and integrate by parts.

Using the identities

$$
\begin{aligned}
& -2 \operatorname{Re} \int_{a}^{b} \hat{v}_{m}^{\prime \prime}(\mu-a) \overline{\hat{v}_{m}^{\prime}} d \mu=-\operatorname{Re} \int_{a}^{b}(\mu-a) d\left|\hat{v}_{m}^{\prime}\right|^{2} \\
& =\left\|\hat{v}_{m}^{\prime}\right\|^{2}-(b-a)\left|\hat{v}_{m}^{\prime}(b)\right|^{2}, \\
& 2 \operatorname{Re} \int_{a}^{b}(\mu-a) \hat{v}_{m} \overline{\hat{v}_{m}^{\prime}} d \mu=\operatorname{Re} \int_{a}^{b}(\mu-a) d\left|\hat{v}_{m}\right|^{2} \\
& =(b-a)\left|\hat{v}_{m}(b)\right|^{2}-\left\|\hat{v}_{m}\right\|^{2}, \\
& -2 \operatorname{Re} \int_{a}^{b}(\mu-a) \hat{v}_{m} \overline{\hat{v}_{m}^{\prime}} \cosh ^{2} \mu d \mu=-\operatorname{Re} \int_{a}^{b}(\mu-a) \cosh ^{2} \mu d\left|\hat{v}_{m}\right|^{2} \\
& =-(b-a) \cosh ^{2} b\left|\hat{v}_{m}(b)\right|^{2}+\left\|\hat{v}_{m}\right\|_{0, \chi}^{2}+\left\|\hat{v}_{m}\right\|_{0, \tilde{\chi}}^{2},
\end{aligned}
$$

and collecting all the real parts, we find

$$
\begin{aligned}
& \left\|\hat{v}_{m}^{\prime}\right\|^{2}+\hat{k}^{2}\left\|\hat{v}_{m}\right\|_{0, \chi}^{2}+\hat{k}^{2}\left\|\hat{v}_{m}\right\|_{0, \tilde{\chi}}^{2}+\lambda_{m}(b-a)\left|\hat{v}_{m}(b)\right|^{2} \\
& =(b-a)\left|\hat{v}_{m}^{\prime}(b)\right|^{2}+\lambda_{m}\left\|\hat{v}_{m}\right\|^{2}+\hat{k}^{2}(b-a) \cosh ^{2} b\left|\hat{v}_{m}(b)\right|^{2} \\
& \quad+2 \operatorname{Re}\left(\hat{g}_{m},(\mu-a) \hat{v}_{m}^{\prime}\right) .
\end{aligned}
$$

Step 3. We now take $\hat{w}_{m}=2 \hat{v}_{m}^{\prime}$ in (6.14). Using the identities

$$
\begin{aligned}
& -2 \operatorname{Re} \int_{a}^{b} \hat{v}_{m}^{\prime \prime} \overline{\hat{v}_{m}^{\prime}} d \mu=\left|\hat{v}_{m}^{\prime}(a)\right|^{2}-\left|\hat{v}_{m}^{\prime}(b)\right|^{2}, \quad 2 \operatorname{Re} \int_{a}^{b} \hat{v}_{m} \overline{\hat{v}_{m}^{\prime}} d \mu=\left|\hat{v}_{m}(b)\right|^{2}, \\
& -2 \operatorname{Re} \int_{a}^{b}\left(\cosh ^{2} \mu\right) \hat{v}_{m} \overline{\hat{v}}_{m}^{\prime} d \mu=-\cosh ^{2} b\left|\hat{v}_{m}(b)\right|^{2}+\left\|\hat{v}_{m}\right\|_{0, \bar{\chi}}^{2},
\end{aligned}
$$

and collecting all the real parts, we get

$\hat{k}^{2}\left\|\hat{v}_{m}\right\|_{0, \bar{\chi}}^{2}+\lambda_{m}\left|\hat{v}_{m}(b)\right|^{2}+\left|\hat{v}_{m}^{\prime}(a)\right|^{2}=\left|\hat{v}_{m}^{\prime}(b)\right|^{2}+\hat{k}^{2} \cosh ^{2} b\left|\hat{v}_{m}(b)\right|^{2}+2 \operatorname{Re}\left(\hat{g}_{m}, \hat{v}_{m}^{\prime}\right)$.

A combination of (6.11), (6.16) and (6.18) leads to

$$
\begin{aligned}
2\left\|\hat{v}_{m}^{\prime}\right\|^{2}+\hat{k}^{2}\left\|\hat{v}_{m}\right\|_{0, \hat{\chi}}^{2}+\left|\hat{v}_{m}^{\prime}(a)\right|^{2}+\lambda_{m}(b-a+1)\left|\hat{v}_{m}(b)\right|^{2}+\frac{1}{2} \tanh b\left|\hat{v}_{m}(b)\right|^{2} \\
\leq \\
\quad(b-a+1)\left|\hat{v}_{m}^{\prime}(b)\right|^{2}+\hat{k}^{2} \cosh ^{2} b(b-a+1)\left|\hat{v}_{m}(b)\right|^{2} \\
\quad+\left|\operatorname{Re}\left(\hat{g}_{m}, \hat{v}_{m}\right)\right|+2\left|\operatorname{Re}\left(\hat{g}_{m},(\mu-a+1) \hat{v}_{m}^{\prime}\right)\right|,
\end{aligned}
$$

where $\hat{\chi}=(\mu-a+1) \sinh (2 \mu)$. Using the boundary condition at $r=b$ in (6.6) yields

$$
\left|\hat{v}_{m}^{\prime}(b)\right|^{2}=\left|\mathcal{T}_{\hat{k}, b}\right|^{2}\left|\hat{v}_{m}(b)\right|^{2}=\left(\hat{k}^{2} \sinh ^{2} b+\frac{1}{4} \tanh ^{2} b\right)\left|\hat{v}_{m}(b)\right|^{2} .
$$

By (6.13) and the Cauchy-Schwarz inequality,

$$
\begin{gathered}
\hat{k}^{2} \cosh (2 b)(b-a+1)\left|\hat{v}_{m}(b)\right|^{2}=\hat{k} \frac{\cosh (2 b)}{\sinh b}(b-a+1)\left|\operatorname{Im}\left(\hat{g}_{m}, \hat{v}_{m}\right)\right| \\
\leq \frac{\hat{k}^{2}}{8}\left\|\hat{v}_{m}\right\|_{0, \hat{\chi}}^{2}+2(b-a+1)^{2} \frac{\cosh ^{2}(2 b)}{\sinh ^{2} b}\left\|\hat{g}_{m}\right\|_{0, \hat{\chi}^{-1}}^{2}
\end{gathered}
$$


and

$$
\begin{gathered}
\frac{1}{4} \tanh ^{2} b(b-a+1)\left|\hat{v}_{m}(b)\right|^{2}=\frac{\tanh ^{2} b}{4 \hat{k} \sinh b}(b-a+1)\left|\operatorname{Im}\left(\hat{g}_{m}, \hat{v}_{m}\right)\right| \\
\leq \frac{\hat{k}^{2}}{8}\left\|\hat{v}_{m}\right\|_{0, \hat{\chi}}^{2}+(b-a+1)^{2} \frac{\tanh ^{4} b}{8 \hat{k}^{4} \sinh ^{2} b}\left\|\hat{g}_{m}\right\|_{0, \hat{\chi}^{-1}}^{2} .
\end{gathered}
$$

On the other hand, using the Cauchy-Schwarz inequality yields

$$
\left|\operatorname{Re}\left(\hat{g}_{m}, \hat{v}_{m}\right)\right| \leq \frac{\hat{k}^{2}}{4}\left\|\hat{v}_{m}\right\|_{0, \hat{\chi}}^{2}+\frac{1}{\hat{k}^{2}}\left\|\hat{g}_{m}\right\|_{0, \hat{\chi}^{-1}}^{2}
$$

and

$$
2\left|\operatorname{Re}\left(\hat{g}_{m},(\mu-a+1) \hat{v}_{m}^{\prime}\right)\right| \leq\left\|\hat{v}_{m}^{\prime}\right\|^{2}+(b-a+1)^{2}\left\|\hat{g}_{m}\right\|^{2} .
$$

Notice that $\cosh ^{2} b+\sinh ^{2} b=\cosh (2 b)$, so the desired result follows from (6.19)(6.23).

6.2. Spectral-Galerkin approximations. Given a cut-off integer $M>0$, we define the Mathieu-Legendre approximation to the solution $v$ of (6.4) by

$$
v_{M N}(\mu, \theta)=\sum_{m=0}^{M} v_{m}^{N}(\mu) \mathrm{me}_{m}(\theta ; q),
$$

where $\left\{v_{m}^{N}\right\}_{m=0}^{M}$ are the solutions of the problem: Find $v_{m}^{N} \in{ }^{0} P_{N}=\left\{\phi \in P_{N}\right.$ : $\phi(a)=0\}$ such that

$$
\begin{aligned}
& \mathbb{B}_{m}\left(v_{m}^{N}, w_{m}^{N}\right)=\left(\partial_{\mu} v_{m}^{N}, \partial_{\mu} w_{m}^{N}\right)+\lambda_{m}\left(v_{m}^{N}, w_{m}^{N}\right) \\
& \quad-\hat{k}^{2}\left(v_{m}^{N}, w_{m}^{N}\right)_{\chi}-\mathcal{T}_{\hat{k}, b} v_{m}^{N}(b) \overline{w_{m}^{N}(b)}=\left(\hat{g}_{m}, w_{m}^{N}\right), \quad \forall w_{m}^{N} \in{ }^{0} P_{N} .
\end{aligned}
$$

As for (6.8), it is clear from the Fredholm Alternative Theorem that (6.25) admits a unique solution. The following result is needed for the error analysis.

Lemma 6.2. If $\hat{g}_{m} \in L_{\tilde{\chi}^{-1}}^{2}(\Lambda)$, we have

$$
\left\|\partial_{\mu} v_{m}^{N}\right\|+\frac{\hat{k}}{2}\left\|v_{m}^{N}\right\|_{0, \tilde{\chi}} \lesssim \widetilde{C}_{a, b, \hat{k}}\left\|\hat{g}_{m}\right\|_{0, \tilde{\chi}^{-1}}
$$

where $\tilde{\chi}=(\mu-a) \sinh (2 \mu)$ and

$$
\widetilde{C}_{a, b, \hat{k}}=\sqrt{2}(b-a)\left[\frac{\cosh (2 b)}{\sinh b}+\frac{\tanh ^{2} b}{4 \hat{k}^{2} \sinh b}+\sqrt{(b-a) \sinh (2 b)}\right]+\frac{1}{\hat{k}} .
$$

Proof. Since the first two test functions $w_{m}^{N}=v_{m}^{N},(\mu-a) \partial_{\mu} v_{m}^{N} \in{ }^{0} P_{N}$, but for the third one $\partial_{\mu} v_{m}^{N} \notin{ }^{0} P_{N}$, the proof of Lemma 6.1 needs to be slightly modified to derive (6.26). For completeness, we sketch the derivation below.

Note that 6.12) - 6.13) hold with $v_{m}^{N}$ in place of $\hat{v}_{m}$. We now take $w_{m}^{N}=$ $2(\mu-a) \partial_{\mu} v_{m}^{N}$ in (6.25), and find that

$$
2 \operatorname{Re}\left(\partial_{\mu} v_{m}^{N}, \partial_{\mu}\left((\mu-a) \overline{\partial_{\mu} v_{m}^{N}}\right)\right)=(b-a)\left|\partial_{\mu} v_{m}^{N}(b)\right|^{2}+\left\|\partial_{\mu} v_{m}^{N}\right\|^{2} .
$$


We also see that the second and third identities in (6.15) hold with $v_{m}^{N}$ in place of $\hat{v}_{m}$. Thus, the real part of (6.25) with $w_{m}^{N}=2(\mu-a) \partial_{\mu} v_{m}^{N}$ becomes

$$
\begin{aligned}
\left\|\partial_{\mu} v_{m}^{N}\right\|^{2}+\hat{k}^{2}\left\|v_{m}^{N}\right\|_{0, \chi}^{2}+\hat{k}^{2}\left\|v_{m}^{N}\right\|_{0, \tilde{\chi}}^{2} & \\
& +(b-a)\left|\partial_{\mu} v_{m}^{N}(b)\right|^{2}+\lambda_{m}(b-a)\left|v_{m}^{N}(b)\right|^{2} \\
= & \lambda_{m}\left\|v_{m}^{N}\right\|^{2}+\hat{k}^{2}(b-a) \cosh ^{2} b\left|v_{m}^{N}(b)\right|^{2}+2 \operatorname{Re}\left(\hat{g}_{m},(\mu-a) \partial_{\mu} v_{m}^{N}\right) \\
& +2(b-a) \operatorname{Re}\left(\mathcal{T}_{\hat{k}, b} v_{m}^{N}(b) \overline{\partial_{\mu} v_{m}^{N}(b)}\right) .
\end{aligned}
$$

Hence, a combination of (6.12) (replacing $\hat{v}_{m}$ by $v_{m}^{N}$ ) and (6.29) leads to

$$
\begin{aligned}
2\left\|\partial_{\mu} v_{m}^{N}\right\|^{2}+\hat{k}^{2}\left\|v_{m}^{N}\right\|_{0, \tilde{\chi}}^{2}+\frac{1}{2} \tanh b\left|v_{m}^{N}(b)\right|^{2} & \\
& +(b-a)\left|\partial_{\mu} v_{m}^{N}(b)\right|^{2}+\lambda_{m}(b-a)\left|v_{m}^{N}(b)\right|^{2} \\
= & \hat{k}^{2}(b-a) \cosh ^{2} b\left|v_{m}^{N}(b)\right|^{2}+2 \operatorname{Re}\left(\hat{g}_{m},(\mu-a) \partial_{\mu} v_{m}^{N}\right)+\operatorname{Re}\left(\hat{g}_{m}, v_{m}^{N}\right) \\
& +2(b-a) \operatorname{Re}\left(\mathcal{T}_{\hat{k}, b} v_{m}^{N}(b) \overline{\partial_{\mu} v_{m}^{N}(b)}\right) .
\end{aligned}
$$

Using the Cauchy-Schwarz inequality yields

$$
\begin{aligned}
2(b-a) & \left|\operatorname{Re}\left(\mathcal{T}_{\hat{k}, b} v_{m}^{N}(b) \overline{\partial_{\mu} v_{m}^{N}(b)}\right)\right| \\
& \leq \frac{b-a}{4}\left|\partial_{\mu} v_{m}^{N}(b)\right|^{2}+(b-a)\left|\mathcal{T}_{\hat{k}, b}\right|^{2}\left|v_{m}^{N}(b)\right|^{2} \\
& \leq \frac{b-a}{4}\left|\partial_{\mu} v_{m}^{N}(b)\right|^{2}+(b-a)\left(\hat{k}^{2} \sinh ^{2} b+\frac{1}{4} \tanh ^{2} b\right)\left|v_{m}^{N}(b)\right|^{2} .
\end{aligned}
$$

Similarly, by (6.13) and the Cauchy-Schwarz inequality,

$$
\begin{gathered}
\hat{k}^{2} \cosh (2 b)(b-a)\left|v_{m}^{N}(b)\right|^{2}=\hat{k} \frac{\cosh (2 b)}{\sinh b}(b-a)\left|\operatorname{Im}\left(\hat{g}_{m}, v_{m}^{N}\right)\right| \\
\leq \frac{\hat{k}^{2}}{8}\left\|v_{m}^{N}\right\|_{0, \tilde{\chi}}^{2}+2(b-a)^{2} \frac{\cosh ^{2}(2 b)}{\sinh ^{2} b}\left\|\hat{g}_{m}\right\|_{0, \tilde{\chi}^{-1}}^{2}
\end{gathered}
$$

and

$$
\begin{gathered}
\frac{1}{4} \tanh ^{2} b(b-a)\left|v_{m}^{N}(b)\right|^{2}=\frac{\tanh ^{2} b}{4 \hat{k} \sinh b}(b-a)\left|\operatorname{Im}\left(\hat{g}_{m}, v_{m}^{N}\right)\right| \\
\leq \frac{\hat{k}^{2}}{8}\left\|\hat{v}_{m}\right\|_{0, \tilde{\chi}}^{2}+(b-a)^{2} \frac{\tanh ^{4} b}{8 \hat{k}^{4} \sinh ^{2} b}\left\|\hat{g}_{m}\right\|_{0, \tilde{\chi}^{-1}}^{2} .
\end{gathered}
$$

On the other hand, using the Cauchy-Schwarz inequality again yields

$$
\left|\operatorname{Re}\left(\hat{g}_{m}, v_{m}^{N}\right)\right| \leq \frac{\hat{k}^{2}}{4}\left\|v_{m}^{N}\right\|_{0, \tilde{\chi}}^{2}+\frac{1}{\hat{k}^{2}}\left\|\hat{g}_{m}\right\|_{0, \tilde{\chi}^{-1}}^{2}
$$

and

$$
\begin{aligned}
& 2\left|\operatorname{Re}\left(\hat{g}_{m},(\mu-a) \partial_{\mu} v_{m}^{N}\right)\right| \leq\left\|\partial_{\mu} v_{m}^{N}\right\|^{2}+\int_{a}^{b}(\mu-a)^{2}\left|\hat{g}_{m}\right|^{2} d \mu \\
& \quad \leq\left\|\partial_{\mu} v_{m}^{N}\right\|^{2}+\int_{a}^{b}(\mu-a)^{3} \sinh (2 \mu) \frac{\left|\hat{g}_{m}\right|^{2}}{(\mu-a) \sinh (2 \mu)} d \mu \\
& \quad \leq\left\|\partial_{\mu} v_{m}^{N}\right\|^{2}+(b-a)^{3} \sinh (2 b)\left\|\hat{g}_{m}\right\|_{0, \tilde{\chi}^{-1}}^{2} .
\end{aligned}
$$

Finally, a combination of (6.30) 
6.3. Error estimations. As in the previous section, we define the space $\widehat{B}^{r}(\Lambda)$ :

$$
\widehat{B}^{r}(\Lambda):=\left\{v \in L^{2}(\Lambda):[(\mu-a)(b-\mu)]^{\frac{k-1}{2}} \partial_{\mu}^{k} v \in L^{2}(\Lambda), \quad 1 \leq k \leq r\right\},
$$

with the norm and semi-norm

$$
\begin{gathered}
\|v\|_{\widehat{B}^{r}(\Lambda)}=\left(\|v\|^{2}+\sum_{k=1}^{r}\left\|[(\mu-a)(b-\mu)]^{\frac{k-1}{2}} \partial_{\mu}^{k} v\right\|^{2}\right)^{\frac{1}{2}}, \\
|v|_{\widehat{B}^{r}(\Lambda)}=\left\|[(\mu-a)(b-\mu)]^{\frac{r-1}{2}} \partial_{\mu}^{r} v\right\| .
\end{gathered}
$$

Let $\varpi(\mu)=(\mu-a)(b-\mu)$. The following approximation result will be used in the error analysis (see Appendix $\mathrm{D}$ for the proof).

Lemma 6.3. There exists a mapping $\pi_{N}^{1}: H^{1}(\Lambda) \rightarrow P_{N}$ satisfying

$$
\pi_{N}^{1} w(a)=w(a), \quad \pi_{N}^{1} w(b)=w(b)
$$

and

$$
\left(\partial_{\mu}\left(\pi_{N}^{1} w-w\right), \partial_{\mu} w_{N}\right)=0, \quad \forall w_{N} \in P_{N} .
$$

Moreover, for any $w \in \widehat{B}^{r}(\Lambda)$ with $r \geq 1$,

$$
\left\|\left(\pi_{N}^{1} w-w\right)^{\prime}\right\|+N\left\|\left(\pi_{N}^{1} w-w\right)\right\|_{0, \varpi^{-1}} \lesssim N^{1-r}|w|_{\widehat{B}^{r}(\Lambda)} .
$$

With the aid of the a priori estimates and the above approximation result, we derive the following error estimates.

Theorem 6.2. Let $\hat{v}_{m}$ and $v_{m}^{N}$ be the solutions of (6.8) and (6.25), respectively. If $\hat{v}_{m} \in{ }^{0} H^{1}(\Lambda) \cap \widehat{B}^{r}(\Lambda)$ with $r \geq 1$, we have

$$
\left\|\partial_{\mu}\left(\hat{v}_{m}-v_{m}^{N}\right)\right\|+\hat{k}\left\|\hat{v}_{m}-v_{m}^{N}\right\|_{0, \tilde{\chi}} \lesssim D_{m, N, \hat{k}} N^{1-r}\left\|\hat{v}_{m}\right\|_{\widehat{B}^{r}(\Lambda)},
$$

where $\tilde{\chi}=(\mu-a) \sinh (2 \mu)$, and

$$
\begin{aligned}
D_{m, N, \hat{k}}:= & {\left[1+(b-a)^{3 / 2} \sinh ^{1 / 2}(2 b) \hat{k} N^{-1}\right.} \\
& \left.+\widetilde{C}_{a, b, \hat{k}}(b-a)^{1 / 2}\left(\lambda_{m}+\frac{\cosh ^{2} b}{(\sinh (2 b))^{1 / 2}} \hat{k}^{2}\right) N^{-1}\right],
\end{aligned}
$$

with $\widetilde{C}_{a, b, \hat{k}}$ being given by (6.27).

Proof. Let $e_{m}^{N}=v_{m}^{N}-\pi_{N}^{1} \hat{v}_{m}$ and $\tilde{e}_{m}^{N}=\hat{v}_{m}-\pi_{N}^{1} \hat{v}_{m}$. By (6.6) and (6.25), we have that $\mathbb{B}_{m}\left(\hat{v}_{m}-v_{m}^{N}, w_{m}^{N}\right)=0$, for all $w_{m}^{N} \in{ }^{0} P_{N}$. Hence, using the fact $\tilde{e}_{m}^{N}(b)=0$ and (6.38) leads to

$$
\mathbb{B}_{m}\left(e_{m}^{N}, w_{m}^{N}\right)=\mathbb{B}_{m}\left(\hat{v}_{m}-\pi_{N}^{1} \hat{v}_{m}, w_{m}^{N}\right)=\lambda_{m}\left(\tilde{e}_{m}^{N}, w_{m}^{N}\right)-\hat{k}^{2}\left(\tilde{e}_{m}^{N} \cosh ^{2} \mu, w_{m}^{N}\right) .
$$

Note that (6.42) can be viewed as (6.25) with $\hat{g}_{m}=\lambda_{m} \tilde{e}_{m}^{N}-\hat{k}^{2}\left(\cosh ^{2} \mu\right) \tilde{e}_{m}^{N}$. Thus, by (6.26),

$$
\left\|\partial_{\mu} e_{m}^{N}\right\|+\frac{\hat{k}}{2}\left\|e_{m}^{N}\right\|_{0, \tilde{\chi}} \leq \widetilde{C}_{a, b, \hat{k}}\left(\lambda_{m}\left\|\tilde{e}_{m}^{N}\right\|_{0, \tilde{\chi}^{-1}}+\hat{k}^{2}\left\|\tilde{e}_{m}^{N}\right\|_{0, \chi^{*}}\right)
$$


where we denote $\chi^{*}=\frac{\cosh ^{4} \mu}{(\mu-a) \sinh (2 \mu)}=\left(\cosh ^{2} \mu\right) \tilde{\chi}^{-1}$. Hence, by Lemma 6.3

$$
\begin{aligned}
\left\|\tilde{e}_{m}^{N}\right\|_{0, \tilde{\chi}^{-1}} & =\frac{\left(b-\nu_{1}\right)^{1 / 2}}{\left(\sinh \left(2 \nu_{1}\right)\right)^{1 / 2}}\left\|\pi_{N}^{1} \hat{v}_{m}-\hat{v}_{m}\right\|_{0, \varpi^{-1}} \\
& \lesssim(b-a)^{1 / 2} N^{-r}\left|\hat{v}_{m}\right|_{\widehat{B}^{r}(\Lambda)}, \\
\left\|\tilde{e}_{m}^{N}\right\|_{0, \chi^{*}} & =\frac{\left(b-\nu_{2}\right)^{1 / 2} \cosh ^{2} \nu_{2}}{\left(\sinh \left(2 \nu_{2}\right)\right)^{1 / 2}}\left\|\pi_{N}^{1} \hat{v}_{m}-\hat{v}_{m}\right\|_{0, \varpi^{-1}} \\
& \lesssim \frac{(b-a)^{1 / 2} \cosh ^{2} b}{(\sinh (2 b))^{1 / 2}} N^{-r}\left|\hat{v}_{m}\right|_{\widehat{B}^{r}(\Lambda)}, \\
\left\|\tilde{e}_{m}^{N}\right\|_{0, \tilde{\chi}}= & \left(\nu_{3}-a\right)\left(b-\nu_{3}\right)^{1 / 2} \sinh ^{1 / 2}\left(2 \nu_{3}\right)\left\|\pi_{N}^{1} \hat{v}_{m}-\hat{v}_{m}\right\|_{0, \varpi^{-1}} \\
& \lesssim(b-a)^{3 / 2} \sin ^{1 / 2}(2 b) N^{-r}\left|\hat{v}_{m}\right|_{\widehat{B}^{r}(\Lambda)},
\end{aligned}
$$

where $\left\{\nu_{i}\right\}_{i=1}^{3}$ are three constants in $(a, b)$.

Next, by the triangle inequality, (6.39) and (6.43) -6.44$)$,

$$
\begin{aligned}
&\left\|\partial_{\mu}\left(\hat{v}_{m}-v_{m}^{N}\right)\right\|+\hat{k}\left\|\hat{v}_{m}-v_{m}^{N}\right\|_{0, \tilde{\chi}} \leq\left\|\partial_{\mu} e_{m}^{N}\right\|+\hat{k}\left\|e_{m}^{N}\right\|_{0, \tilde{\chi}}+\left\|\partial_{\mu} \tilde{e}_{m}^{N}\right\|+\hat{k}\left\|\tilde{e}_{m}^{N}\right\|_{0, \tilde{\chi}} \\
& \lesssim {\left[1+(b-a)^{3 / 2} \sinh ^{1 / 2}(2 b) \hat{k} N^{-1}\right.} \\
&\left.+\widetilde{C}_{a, b, \hat{k}}(b-a)^{1 / 2}\left(\lambda_{m}+\frac{\cosh ^{2} b}{(\sinh (2 b))^{1 / 2}} \hat{k}^{2}\right) N^{-1}\right] N^{1-r}\left|\hat{v}_{m}\right|_{\widehat{B}^{r}(\Lambda)} .
\end{aligned}
$$

This ends the proof.

Remark 6.1. To illustrate how the error $\hat{v}_{m}-v_{m}^{N}$ behaves with respect to $N, k$ and $b$ with fixed $a>0$ and $m \geq 0$, we consider a typical oscillatory function $\hat{v}_{m}(\mu)=e^{\mathrm{i} k \mu}$ to be the solution of (6.8). Then, for any $r>0$,

$$
\begin{aligned}
\left|\hat{v}_{m}\right|_{\widehat{B}^{r}(\Lambda)}^{2} & =\int_{a}^{b}\left|\partial_{\mu}^{r} \hat{v}_{m}\right|^{2}((\mu-a)(b-\mu))^{r-1} d \mu \\
& \leq k^{2 r} \int_{a}^{b}((\mu-a)(b-\mu))^{r-1} d \mu \lesssim k\left(k \frac{b-a}{2}\right)^{2 r-1} .
\end{aligned}
$$

Notice that $D_{m, N, \hat{k}} \lesssim 1+(b-a)^{3 / 2} \cosh b k^{2} N^{-1}$. Therefore, Theorem6.2 indicates that for this typical solution, we have that for any $r \geq 1$,

$\left\|\partial_{\mu}\left(\hat{v}_{m}-v_{m}^{N}\right)\right\|+\hat{k}\left\|\hat{v}_{m}-v_{m}^{N}\right\| \lesssim\left(1+(b-a)^{3 / 2} \cosh b k^{2} N^{-1}\right) k \sqrt{\frac{b-a}{2}}\left(\frac{k(b-a)}{2 N}\right)^{r-1}$.

Hence, the error will decay exponentially as soon as $\frac{k(b-a)}{2 N}<1$. Therefore, we can significantly reduce the computational cost by choosing $b$ closer to $a$.

We now present some numerical results. We solved (6.25) with the function $\hat{g}_{m}$ such that the exact solution is $\hat{v}_{m}=\exp (\mathrm{i} k \mu)$. We refer to [12] for more details on the actual implementation of (6.25). In Figure 6.1, we plot numerical results which depict the convergence behaviors of our numerical scheme with $(b-a) / 2=1$. On the left, we fix $k=100$ and plot the $L^{2}$-errors with respect to $N$. We observe that the error decays exponentially fast as soon as $N>k$. Next, we denote $\alpha=\frac{k(b-a)}{2 N}$ and plot on the right of Figure 6.1 the $L^{2}$-errors with three different $\alpha$. We observe that in all three cases, the error converges exponentially. 

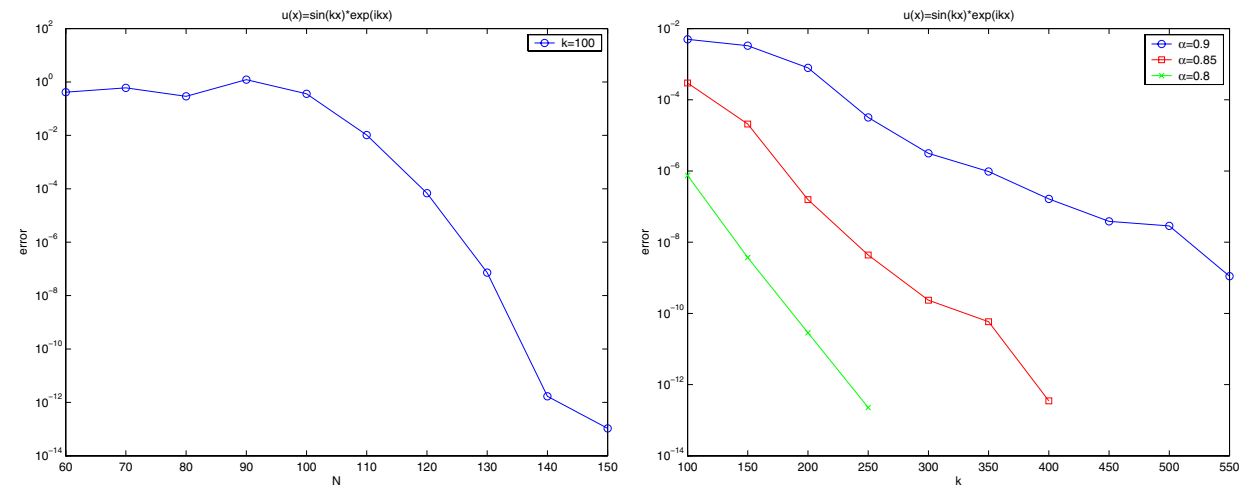

FiguRE 6.1. Left: $L^{2}$-error with respect to $N$ for $k=100$. Right: $L^{2}$-errors with three different $\alpha$ where $\alpha=\frac{k(b-a)}{2 N}$.

We now estimate the error of the Legendre-Mathieu spectral approximation. Let $Q=\Lambda \times I=(a, b) \times(0,2 \pi)$, and introduce the space

$$
\widehat{H}_{p}^{r, s}(Q):=L^{2}\left(I ; \widehat{B}^{r}(\Lambda)\right) \cap H_{p}^{s-1}\left(I ;{ }^{0} H^{1}(\Lambda)\right) \cap H_{p}^{s}\left(I ; L^{2}(\Lambda)\right), \quad r, s \geq 1,
$$

with the norm

$$
\|v\|_{\widehat{H}_{p}^{r, s}(Q)}=\left(\sum_{m=0}^{\infty}\left[\left|\hat{v}_{m}\right|_{\widehat{B}^{r}(\Lambda)}^{2}+\lambda_{m}^{s-1}\left\|\partial_{\mu} \hat{v}_{m}\right\|_{L^{2}(\Lambda)}^{2}+\lambda_{m}^{s}\left\|\hat{v}_{m}\right\|_{L^{2}(\Lambda)}^{2}\right]\right)^{\frac{1}{2}},
$$

where $\left\{\hat{v}_{m}\right\}$ are the coefficients of the Mathieu expansion of $v$.

Theorem 6.3. Let $v$ be the solution of (6.4), and let $v_{M N}$ be the approximate solution given by (6.24) - (6.25). Then, for $v \in \widehat{H}_{p}^{r, s}(Q)$ with $r, s \geq 1$, we have

$$
\begin{aligned}
& \left\|v-v_{M N}\right\|_{L_{\bar{\chi}}^{2}(Q)} \lesssim\left(D_{M, N, \hat{k}} \hat{k}^{-1} N^{1-r}+\lambda_{M+1}^{-s / 2}\right)\|v\|_{\widehat{H}_{p}^{r, s}(Q)}, \\
& \left\|\partial_{\mu}\left(v-v_{M N}\right)\right\|_{L^{2}(Q)} \lesssim\left(D_{M, N, \hat{k}} N^{1-r}+\lambda_{M+1}^{(1-s) / 2}\right)\|v\|_{\widehat{H}_{p}^{r, s}(Q)},
\end{aligned}
$$

and

$$
\left\|\partial_{\theta}\left(v-v_{M N}\right)\right\|_{L^{2}(Q)} \lesssim\left((b-a) D_{M, N, \hat{k}} \lambda_{M}^{1 / 2} N^{1-r}+\lambda_{M+1}^{(1-s) / 2}\right)\|v\|_{\widehat{H}_{p}^{r, s}(Q)},
$$

where $\tilde{\chi}=(\mu-a) \sinh (2 \mu), D_{M, N, \hat{k}}$ is given in (6.41), and $\lambda_{m}$ is the eigenvalue of the Mathieu equation (cf. (3.3)).

Proof. We derive from the orthogonality of the Mathieu functions and (6.40) that

$$
\begin{aligned}
\| v & -v_{M N}\left\|_{L_{\tilde{\chi}}^{2}(Q)}^{2}=\sum_{m=0}^{M} \pi\right\| \hat{v}_{m}-v_{m}^{N}\left\|_{L_{\tilde{\chi}}^{2}(\Lambda)}^{2}+\sum_{m=M+1}^{\infty} \pi\right\| \hat{v}_{m} \|_{L_{\tilde{\chi}}^{2}(\Lambda)}^{2} \\
& \leq \sum_{m=0}^{M} \pi\left\|\hat{v}_{m}-v_{m}^{N}\right\|_{L_{\tilde{\chi}}^{2}(\Lambda)}^{2}+\lambda_{M+1}^{-s} \sum_{m=M+1}^{\infty} \pi \lambda_{m}^{s}\left\|\hat{v}_{m}\right\|_{L_{\tilde{\chi}}^{2}(\Lambda)}^{2} \\
& \lesssim \hat{k}^{-2} D_{M, N, \hat{k}}^{2} N^{2-2 r} \sum_{m=0}^{M}\left|\hat{v}_{m}\right|_{\hat{B}^{r}(\Lambda)}^{2}+\lambda_{M+1}^{-s} \sum_{m=M+1}^{\infty} \lambda_{m}^{s}\left\|\hat{v}_{m}\right\|_{L_{\tilde{\chi}}^{2}(\Lambda)}^{2} .
\end{aligned}
$$


Similarly, using (6.40) leads to

$$
\begin{aligned}
\left\|\partial_{\mu}\left(v-v_{M N}\right)\right\|_{L^{2}(Q)}^{2} \lesssim & D_{M, N, \hat{k}}^{2} N^{2-2 r} \sum_{m=0}^{M}\left|\hat{v}_{m}\right|_{\widehat{B}^{r}(\Lambda)}^{2} \\
& +\lambda_{M+1}^{1-s} \sum_{m=M+1}^{\infty} \lambda_{m}^{s-1}\left\|\partial_{\mu} \hat{v}_{m}\right\|_{L^{2}(\Lambda)}^{2} .
\end{aligned}
$$

We now prove (6.50). One verifies, by using the Hardy inequality (B.3), that for any $w \in{ }^{0} H^{1}(\Lambda)$,

Hence, by (6.40),

$$
\|w\|_{L^{2}(\Lambda)} \leq 2(b-a)\left\|w^{\prime}\right\|_{L^{2}(\Lambda)}
$$

$$
\left\|\hat{v}_{m}-v_{m}^{N}\right\|_{L^{2}(\Lambda)} \lesssim(b-a)\left\|\partial_{\mu}\left(\hat{v}_{m}-v_{m}^{N}\right)\right\|_{L^{2}(\Lambda)} \lesssim(b-a) D_{m, N, \hat{k}} N^{1-r}\left|\hat{v}_{m}\right|_{\widehat{B}^{r}(\Lambda)} .
$$

By (3.10)-(3.11), (6.5) and (6.24), we have from (6.52) that

$$
\begin{aligned}
& \left\|\partial_{\theta}\left(v-v_{M N}\right)\right\|^{2}+\hat{k}^{2}\left\|\left(v-v_{M N}\right) \cos \theta\right\|^{2} \\
& \quad=\sum_{m=0}^{M} \pi \lambda_{m}\left\|\hat{v}_{m}-v_{m}^{N}\right\|_{L^{2}(\Lambda)}^{2}+\sum_{m=M+1}^{\infty} \pi \lambda_{m}\left\|\hat{v}_{m}\right\|_{L^{2}(\Lambda)}^{2} \\
& \quad \lesssim(b-a)^{2} \lambda_{M} D_{M, N, \hat{k}}^{2} N^{2-2 s} \sum_{m=0}^{M}\left|\hat{v}_{m}\right|_{\widehat{B}^{r}(\Lambda)}^{2}+\lambda_{M+1}^{1-s} \sum_{m=M+1}^{\infty} \lambda_{m}^{s}\left|\hat{v}_{m}\right|_{L^{2}(\Lambda)}^{2} .
\end{aligned}
$$

This ends the proof.

\section{Concluding Remarks}

The Mathieu functions are classical special functions which are important tools for solving certain partial differential equations in elliptic domains. In particular, they enable us to reduce the Helmholtz or the modified Helmholtz equation in a twodimensional elliptic domain to a sequence of one-dimensional equations that are easy to solve numerically. However, to the best of our knowledge, there was no rigorous approximation result available for Mathieu expansions. This paper established a first set of such approximation results which will serve as basic ingredients for the numerical analysis of Mathieu approximations to PDEs.

More precisely, we developed optimal approximation results for the angular Mathieu functions. These approximation results are very similar to those for the Fourier expansions. Namely, the orthogonal projection based on Mathieu expansions of a periodic function converges in the same way as that based on Fourier expansions.

As examples of applications, we applied the approximation results developed in this paper to establish optimal error estimates for the Mathieu-Legendre approximation to the modified Helmholtz equation and the Helmholtz equation. We also presented illustrative numerical results which are consistent with our theoretical analysis.

\section{Appendix A. ReCURRence Relations}

The coefficients $\left\{A_{j}^{(n)}, B_{j}^{(n)}\right\}$ in the expansion (2.10) satisfy the following recursive relations: 
- Even functions of period $\pi$ (i.e., $\mathrm{ce}_{2 m}$ and $n=2 m$ ):

$$
\left(a_{n}-j^{2}\right) A_{j}^{(n)}-q\left(A_{j-2}^{(n)}+A_{j+2}^{(n)}\right)=0, \quad j \geq 3,
$$

and

$$
a_{n} A_{0}^{(n)}-q A_{2}^{(n)}=0, \quad\left(a_{n}-4\right) A_{2}^{(n)}-q\left(A_{0}^{(n)}+A_{4}^{(n)}\right)=0 .
$$

- Even functions of period $2 \pi$ (i.e., $\mathrm{ce}_{2 m+1}$ and $n=2 m+1$ ):

$$
\left(a_{n}-1\right) A_{1}^{(n)}-q\left(A_{1}^{(n)}+A_{3}^{(n)}\right)=0,
$$

along with A.1 for $j \geq 3$.

- Odd functions of period $\pi$ (i.e., $\mathrm{se}_{2 m}$ and $n=2 m$ ):

$$
\left(b_{n}-j^{2}\right) B_{j}^{(n)}-q\left(B_{j-2}^{(n)}+B_{j+2}^{(n)}\right)=0, \quad j \geq 3,
$$

and

$$
\left(b_{n}-4\right) B_{2}^{(n)}-q B_{4}^{(n)}=0 .
$$

- Odd functions of period $2 \pi$ (i.e., $\mathrm{se}_{2 m+1}$ and $n=2 m+1$ ):

$$
\left(b_{n}-1\right) B_{1}^{(n)}+q\left(B_{1}^{(n)}-B_{3}^{(n)}\right)=0,
$$

along with A.1 for $j \geq 3$.

\section{Appendix B. An embedding inequality}

We have the following inequality:

$$
\|w\| \leq 2\left\|w^{\prime}\right\|, \quad \forall w \in H^{1}(0,1) \text { with } w(1)=0 .
$$

Proof. We recall Hardy's inequality ([20])

$$
\int_{a}^{b}\left[\int_{\mu}^{b} \phi(\nu) d \nu\right]^{2}(b-\mu)^{d-2} d \mu \leq \frac{4}{1-d} \int_{a}^{b} \phi^{2}(\mu)(b-\mu)^{d} d \mu, \quad d<1
$$

and

$$
\int_{a}^{b}\left[\int_{a}^{\mu} \phi(\nu) d \nu\right]^{2}(\mu-a)^{d-2} d \mu \leq \frac{4}{1-d} \int_{a}^{b} \phi^{2}(\mu)(\mu-a)^{d} d \mu, \quad d<1 .
$$

Thanks to $w(1)=0$, taking $\phi=w^{\prime}, a=0, b=1$ and $d=0$ in (‥2) leads to

$$
\|w\|^{2} \leq \int_{0}^{1}|w|^{2}(1-\mu)^{-2} d \mu \leq 4\left\|w^{\prime}\right\|^{2}
$$

This ends the proof.

\section{Appendix C. Proof of Lemma 5.1}

Taking $v_{m}=\hat{u}_{m}$ in (5.13)-(5.14), we use the Cauchy-Schwarz inequality and the embedding inequality (B.1) that

$$
\begin{aligned}
\left\|\hat{u}_{m}^{\prime}\right\|^{2}+\lambda_{m}\left\|\hat{u}_{m}\right\|^{2} & +4 \rho\left\|\hat{u}_{m}\right\|_{0, \chi}^{2} \leq\left|\left(\hat{f}_{m}, \hat{u}_{m}\right)\right| \\
& \leq 2\left\|\hat{f}_{m}\right\|\left\|\hat{u}_{m}^{\prime}\right\| \leq \frac{1}{2}\left\|\hat{u}_{m}^{\prime}\right\|^{2}+2\left\|\hat{f}_{m}\right\|^{2} .
\end{aligned}
$$


We verify by integration by parts and the fact $\hat{u}_{m}^{\prime}(0)=\hat{u}_{m}(1)=0$ that

$$
\begin{gathered}
-\int_{0}^{1} \hat{u}_{m} \hat{u}_{m}^{\prime \prime} \cosh ^{2} \mu d \mu=\int_{0}^{1}\left|\hat{u}_{m}^{\prime}\right|^{2} \cosh ^{2} \mu d \mu+\int_{0}^{1} \hat{u}_{m} \hat{u}_{m}^{\prime} \sinh (2 \mu) d \mu \\
=\left\|\hat{u}_{m}^{\prime}\right\|_{0, \chi}^{2}+\frac{1}{2}\left|\hat{u}_{m}(1)\right|^{2} \sinh (2)-\int_{0}^{1}\left|\hat{u}_{m}\right|^{2} \cosh (2 \mu) d \mu \\
=\left\|\hat{u}_{m}^{\prime}\right\|_{0, \chi}^{2}+\frac{1}{2}\left|\hat{u}_{m}(1)\right|^{2} \sinh (2)+\left\|\hat{u}_{m}\right\|^{2}-2\left\|\hat{u}_{m}\right\|_{0, \chi}^{2} .
\end{gathered}
$$

Multiplying the first equation in (5.12) by $-\hat{u}_{m}^{\prime \prime}$ and integrating the resulting equation over $(0,1)$, we derive from a direct calculation that

$$
\begin{aligned}
\left\|\hat{u}_{m}^{\prime \prime}\right\|^{2} & +\lambda_{m}\left\|\hat{u}_{m}^{\prime}\right\|^{2}+4 \rho\left\|\hat{u}_{m}^{\prime}\right\|_{0, \chi}^{2}+2 \rho\left|\hat{u}_{m}(1)\right|^{2} \sinh (2)+4 \rho\left\|\hat{u}_{m}\right\|^{2} \\
& \leq 8 \rho\left\|\hat{u}_{m}\right\|_{0, \chi}^{2}+\left|\left(\hat{f}_{m}, \hat{u}_{m}^{\prime \prime}\right)\right| \leq 8 \rho\left\|\hat{u}_{m}\right\|_{0, \chi}^{2}+\frac{1}{2}\left\|\hat{f}_{m}\right\|^{2}+\frac{1}{2}\left\|\hat{u}_{m}^{\prime \prime}\right\|^{2} .
\end{aligned}
$$

Since by (C.1), $8 \rho\left\|\hat{u}_{m}\right\|_{0, \chi}^{2} \leq 4\left\|\hat{f}_{m}\right\|^{2}$, a combination of (C.1) and (C.3) leads to

$$
\left\|\hat{u}_{m}\right\|_{2}^{2}+\lambda_{m}\left\|\hat{u}_{m}\right\|_{1}^{2} \lesssim\left\|\hat{f}_{m}\right\|^{2} .
$$

Next, by the first equation of (6.6) and (C.4),

$$
\lambda_{m}\left\|\hat{u}_{m}\right\| \leq\left\|\hat{u}_{m}^{\prime \prime}\right\|+4 \rho\left\|\cosh ^{2} \mu \hat{u}_{m}\right\|+\left\|\hat{f}_{m}\right\| \lesssim\left\|\hat{f}_{m}\right\|,
$$

which completes the proof of Lemma 5.1

\section{Appendix D. Proof of Lemma 6.3}

We first recall a generalized Jacobi approximation (with a change variable from $(-1,1)$ to $\Lambda=(a, b)$ and an extension to complex-valued functions) with parameters $\alpha=\beta=-1$ (cf. Theorem 3.1 of [15]):

Let $\varpi(\mu)=(\mu-a)(b-\mu)$, and $\pi_{N}^{-1}$ be the orthogonal projection from $L_{\varpi-1}^{2}(\Lambda)$ onto $P_{N}^{0}=H_{0}^{1}(\Lambda) \cap P_{N}$ such that

$$
\int_{a}^{b}\left(\pi_{N}^{-1} v-v\right) \overline{v_{N}} \varpi^{-1}(\mu) d \mu=0, \quad \forall v_{N} \in P_{N}^{0} .
$$

Then for any $v \in L_{\varpi^{-1}}^{2}(\Lambda) \cap \widehat{B}^{r}(\Lambda)$ with $r \geq 1$, we have

$$
\left\|\partial_{\mu}\left(\pi_{N}^{-1} v-v\right)\right\|+N\left\|\pi_{N}^{-1} v-v\right\|_{0, \varpi^{-1}} \lesssim N^{1-r}|v|_{\widehat{B}^{r}(\Lambda)} .
$$

Next, using the Hardy inequalities (B.2) $-(\mathrm{B} .3)$ and an argument similar to that in Appendix B, we have $H_{0}^{1}(\Lambda) \subseteq L_{\varpi^{-1}}^{2}(\Lambda)$. Moreover, we find from [15] (see (2.5)) that $\pi_{N}^{-1}$ is also the $H_{0}^{1}(\Lambda)$-orthogonal projection, namely,

$$
\int_{a}^{b}\left(\pi_{N}^{-1} v-v\right)^{\prime} \overline{v_{N}^{\prime}} d \mu=0, \quad \forall v_{N} \in P_{N}^{0}
$$

For any $w \in H^{1}(\Lambda)$, let $w^{*}(\mu)$ be the linear interpolation of $w$ associated with the points $\mu=a, b$, i.e.,

$$
w^{*}(\mu)=\frac{\mu-b}{a-b} w(a)+\frac{\mu-a}{b-a} w(b) \in P_{1} .
$$

It is clear that $w-w^{*} \in H_{0}^{1}(\Lambda) \subseteq L_{\varpi^{-1}}^{2}(\Lambda)$. Now, we define

$$
\pi_{N}^{1} w=\pi_{N}^{-1}\left(w-w^{*}\right)+w^{*} \in P_{N}, \quad \forall w \in H^{1}(\Lambda) .
$$


Obviously, $\pi_{N}^{1} w$ satisfies (6.37). Next we verify (6.38). For this purpose, let $w_{N}^{*}$ be the linear interpolation function of $w_{N}$ as defined in (D.3). We derive from (D.2) and integration by parts that for any $w_{N} \in P_{N}$,

$$
\begin{aligned}
\int_{a}^{b} \partial_{\mu} & \left(\pi_{N}^{1} w-w\right) \overline{\partial_{\mu} w_{N}} d \mu \\
\quad & =\int_{a}^{b} \partial_{\mu}\left(\pi_{N}^{-1}\left(w-w^{*}\right)-\left(w-w^{*}\right)\right) \overline{\partial_{\mu}\left(\left(w_{N}-w_{N}^{*}\right)+w_{N}^{*}\right)} d \mu \\
& =\int_{a}^{b} \partial_{\mu}\left(\pi_{N}^{-1}\left(w-w^{*}\right)-\left(w-w^{*}\right)\right) \overline{\partial_{\mu} w_{N}^{*}} d \mu \\
& =\left.\frac{w_{N}^{*}(b)-w_{N}^{*}(a)}{b-a}\left(\pi_{N}^{-1}\left(w-w^{*}\right)-\left(w-w^{*}\right)\right)\right|_{a} ^{b}=0 .
\end{aligned}
$$

Hence, (6.38) holds. Next, by (D.1),

$$
\left\|\partial_{\mu}\left(\pi_{N}^{1} w-w\right)\right\|+N\left\|\pi_{N}^{1} w-w\right\|_{0, \varpi^{-1}} \lesssim N^{1-r}\left|w-w^{*}\right|_{\widehat{B}^{r}(\Lambda)} .
$$

Since $\partial_{\mu}^{r} w^{*}=0$ for $r \geq 2$, and for $r=1$,

$$
\left\|\partial_{\mu} w^{*}\right\|=(b-a)^{-1 / 2}|w(b)-w(a)| \leq \frac{1}{\sqrt{b-a}} \int_{a}^{b}\left|\partial_{\mu} w\right| d \mu \leq\left\|\partial_{\mu} w\right\|
$$

we have $\left|w-w^{*}\right|_{\widehat{B}^{r}(\Lambda)} \leq 2|w|_{\widehat{B}^{r}(\Lambda)}$, which, together with (D.5), leads to the desired result.

\section{ACKNOWLEDGEMENT}

The authors would like to thank Mr. Qirong Fang for performing the numerical computations presented in Section 6.

\section{REFERENCES}

[1] M. Abramowitz and I. Stegun. Handbook of Mathematical functions. Dover, New York, 1964.

[2] R. A. Adams. Sobolev spaces. Acadmic Press, New York, 1975. MR0450957 (56:9247)

[3] F. A. Alhargan. Algorithm for the computation of all Mathieu functions of integer orders. ACM Trans. Math. Software, 26:390-407, 2001.

[4] F. A. Alhargan and S. R. Judah. Frequency response characteristics of multiport planar elliptic patch. IEEE Trans. Microwave Theory Tech., MIT-40:1726-1730, 1992.

[5] F. A. Alhargan and S. R. Judah. Mode charts for confocal annular elliptic resonators. IEE Proc-Microwave Antennas Propag., 143(4):358-360, 1996.

[6] Fayez A. Alhargan. A complete method for the computations of Mathieu characteristic numbers of integer orders. SIAM Rev., 38(2):239-255, 1996. MR1391228 (97h:33036)

[7] E. T. Whittaker amd G. N. Watson. A course of modern analysis. Cambridge University Press, 4th edition, 1927. MR1424469 (97k:01072)

[8] M. Baeva, P. Baev, and A. Kaplan. An analysis of the heat transfer from a moving elliptical cylinder. J. Phys. D: Appl. Phys., 30:1190-1196, 1997.

[9] J. P. Boyd and H. Ma. Numerical study of elliptical modons using a spectral method. J. Fluid Mech., 221:597-611, 1990.

[10] C. Canuto, M. Y. Hussaini, A. Quarteroni, and T. A. Zang. Spectral methods. Scientific Computation. Springer-Verlag, Berlin, 2006. Fundamentals in single domains. MR 2223552 (2007c:65001)

[11] R. Courant and D. Hilbert. Methods of Mathematical Physics, volume 1. Interscience Publishers, 1953. MR0065391(16:426a)

[12] Qirong Fang, Jie Shen, and Li-Lian Wang. A stable and high-order method for acoustic scattering exterior to a two-dimensional elongated domain. Submitted to J. Comput. Phys.

[13] D. Funaro. Polynomial approxiamtions of differential equations. Springer-Verlag, 1992. MR:1176949(94c:65078) 
[14] Marcus J. Grote and Joseph B. Keller. On nonreflecting boundary conditions. J. Comput. Phys., 122(2):231-243, 1995. MR1365434 (96j:65142)

[15] Ben-Yu Guo, Jie Shen, and Li-Lian Wang. Optimal spectral-Galerkin methods using generalized Jacobi polynomials. J. Sci. Comput., 27(1-3):305-322, 2006. MR2285783 (2008f:65233)

[16] Ben-yu Guo and Li-Lian Wang. Jacobi approximations in non-uniformly Jacobi-weighted Sobolev spaces. J. Approx. Theory, 128(1):1-41, 2004. MR2063010 (2005h:41010)

[17] J. C. Gutierrez-Vega, M. D. Iturbe-Castillo, and S. Chavez-Cerda. Alternative formulation for invariant optical fields: Mathieu beams. Opt. Lett., 25(20):1493-1495, 2000.

[18] J. C. Gutierrez-Vega, R. M. Rodriguez-Dagnino, M. A. Menesses-Nava, and S. ChavezCerda. Mathieu functions, a visual approach. Am. J. Phys., 71(3):233-242, 2003.

[19] T. M. Habashy, J. A. Kong, and W. C. Chew. Scalar and vector Mathieu transform pairs. J. Appl. Phys., 60:3395-3399, 1986.

[20] G. H. Hardy, J. E. Littlewood, and G. Polya. Inequalities. Cambridge University, Cambridge, UK, 1952. MR0046395 (13:727e)

[21] R. Holland and V. P. Cable. Mathieu functions and their applications to scattering by a coated strip. IEEE Trans. Electromagnetic Compatibility, EC-34:9-16, 1992.

[22] Frank Ihlenburg. Finite element analysis of acoustic scattering, volume 132 of Applied Mathematical Sciences. Springer-Verlag, New York, 1998. MR1639879 (99g:65114)

[23] C. Pask J. D. Love and C. Winkler. Rays and modes on step-index multimode elliptical waveguides. IEE J. Microwaves, Optics and Acoustics, 3:231-238, 1979.

[24] Ming-Chih Lai. Fast direct solver for Poisson equation in a 2D elliptical domain. Numer. Methods Partial Differential Equations, 20(1):72-81, 2004. MR2020251(2004j:65175)

[25] J. E. Lewis and G. Deshpande. Models on elliptical cross-section dielectric-tube waveguides. IEE J. Microwaves, Optics and Acoustics, 3:112-117, 1979.

[26] A. Linder and H. Freese. A new method to compute Mathieu functions. J. Phys. A, 27:55655571, 1994. MR 1295380 (95j:33058)

[27] E. Mathieu. Le mouvement vibratoire d'une membrane de forme elliptique. J. Math. Pures Appl., 13:137-203, 1868.

[28] N. W. McLachlan. Theory and applications of Mathieu functions. Oxford Press, London, 1951. MR 0021158 (9:31b)

[29] J. Meixner and F. W. Schäfke. Mathieusche funktionen und sphäoidfunktioner. Springer, Berlin, 1954. MR 0066500 (16:586g)

[30] R. Mittal and S. Balachandar. Direct numerical simulation of flow past elliptic cylinders. J. Comput. Phys., 124:351-367, 1996.

[31] Jean-Claude Nédélec. Acoustic and electromagnetic equations, volume 144 of Applied Mathematical Sciences. Springer-Verlag, New York, 2001. Integral representations for harmonic problems. MR 1822275 (2002c:35003)

[32] L. Ruby. Applications of the Mathieu equation. Am. J. Phys., 64:39-44, 1996. MR1366704 (96h:34097)

[33] Zhang Shangjie and Jin Jianming. Computation of special functions. John Wiley, 1996. MR:1406797 (97m:65001)

[34] Jie Shen and Li-Lian Wang. Spectral approximation of the Helmholtz equation with high wave numbers. SIAM J. Numer. Anal., 43(2):623-644 (electronic), 2005. MR2177883 (2006j:65375)

[35] Roger Temam. Infinite-dimensional dynamical systems in mechanics and physics, volume 68 of Applied Mathematical Sciences. Springer-Verlag, New York, 1988. MR953967 (89m:58056)

[36] N. Toyama and K. Shogen. Computation of the value of the even and the odd Mathieu funcitons of order $n$ for a given parameter $s$ and an argument $x$. IEEE Trans. Ant. and Propagat., 32(5):537-539, 1994. MR.748375 (85j:33006)

Department of Mathematics, Purdue University, West Lafayette, Indiana 47907

E-mail address: shen@math.purdue.edu

Division of Mathematical Sciences, School of Physical and Mathematical Sciences, Nanyang Technological University, 637616, Singapore

E-mail address: lilian@ntu.edu.sg 